\title{
Dye-loaded nanoemulsions: biomimetic fluorescent nanocarriers for bioimaging and nanomedicine
}

\author{
Andrey S. Klymchenko ${ }^{1, \S,}$ Fei Liu, ${ }^{1,2,}$ Mayeul Collot, ${ }^{1}$ Nicolas Anton, ${ }^{2}$ \\ ${ }^{1}$ Laboratory of Biophotonic and Pathologies, CNRS UMR 7021, Université de Strasbourg, \\ Faculté de Pharmacie, 74, Route du Rhin, 67401 Illkirch, France \\ ${ }^{2}$ Université de Strasbourg, CNRS, CAMB UMR 7199, F-67000 Strasbourg, France \\ $\S$ These authors contributed equally to this work. \\ Corresponding author: andrey.klymchenko@unistra.fr
}

Lipid nanoemulsions (NEs), owing to their controllable size (20 to $500 \mathrm{~nm}$ ), stability and bio-compatibility, are now frequently used in various fields, such as food, cosmetics, pharmaceuticals, drug delivery, and even as nano-reactor for chemical synthesis. Moreover, being composed of components generally recognized as safe (GRAS), they can be considered as "green" nanoparticles that mimic closely lipoproteins and intracellular lipid droplets. Therefore, they attracted attention as carriers of drugs and fluorescent dyes for both bioimaging and studying the fate of nanoemulsions in cells and small animals. In this review, we describe the composition of dye-loaded NEs, methods for their preparation and emerging biological applications. We focus on design of bright fluorescent NEs with high dye loading and minimal aggregation-caused quenching (ACQ). Common issues including dye leakage and NEs stability are discussed, highlighting advanced techniques for their characterization, such as Förster resonance energy transfer (FRET) and fluorescence correlation spectroscopy (FCS). Attempts to functionalize NEs surface are also discussed. Then, biological applications for bioimaging and single-particle tracking in cell and small animals as well as biomedical applications for photodynamic therapy are described. Finally, challenges and future perspectives of fluorescent NEs are discussed.

Keywords: fluorescent nanoemulsions; lipophilic dyes; dye leakage; in vitro and in vivo imaging; passive and active targeting. 


\section{Introduction}

In the past decade, a tremendous effort has been done for designing fluorescent (more general term luminescent) nanomaterials for bioimaging and biomedical applications. ${ }^{[1,2]}$ On one hand, fluorescent nanoparticles (NPs) are direct analogues of drug nanocarriers that can be readily tracked in cells and live animals for better understanding the mechanisms and the bottlenecks in the field of drug delivery. On the other hand, fluorescent NPs are attractive because they can be much brighter than fluorescent dye molecules, which is of key importance for improving resolution and sensitivity of fluorescence detection and imaging. ${ }^{[3,4]}$ The controlled size of NPs, ranging from 10 to $200 \mathrm{~nm}$, can tune drastically their biodistribution in vivo and capacity to target pathological tissues, such as tumors. ${ }^{[5]}$ Finally, versatile surface chemistry of NPs allows minimizing their non-specific off-target interactions and enable grafting specific ligands for targeting the cells of interest. ${ }^{[6,7]}$

NPs can be classified based on chemical composition into inorganic and organic ones. Typical examples of luminescent inorganic NPs include semiconductor quantum dots (QDs), ${ }^{[8,9]}$ dye-doped silica NPs,${ }^{[10,11]}$ and upconverting NPs. ${ }^{[12,13]}$ QDs dominated the field of luminescent NPs for bioimaging applications since 1990s because of important advantages as point-like emissive sources: ${ }^{[14]}$ their tunable and narrow emission band and continuous absorption spectrum as well as exceptional photostability, resisting to prolonged irradiation. However, they also revealed a number of drawbacks, such as fluorescence intermittency, or blinking, and their challenging surface chemistry that requires protection of the inorganic core from aqueous solution by relatively thick organic biocompatible shell. However, the key problem is their highly toxic elements, such as $\mathrm{Cd}$ and Se, along with the lack of biodegradability and accumulation in the body. These issues raise questions on their long-term toxicity and severely limit their future applications in nanomedicine (unless they are replaced with less toxic components). ${ }^{[15-17]}$ Similar problems should be addressed in biological applications of upconverting NPs. ${ }^{[18]}$

NPs based on organic compounds open the gate to a "green" approach in nanotechnology, where they can be fabricated from natural and/or biodegradable components. Fluorescence can be readily implemented into these NPs by encapsulating a specially designed fluorescent dyes, providing superior control optical properties of NPs, such as color and brightness. ${ }^{[19]}$ The current fluorescent organic nanocarriers are dominated by conjugated polymer NPs ${ }^{[20,21]}$ and dye-loaded polymer NPs. ${ }^{[3,22,23]}$ However, in the biomedical context, the use of polymers raises some issues, related to the residues of monomers and solvents after their synthesis and formulation of NPs as well as polymer toxicity. Moreover, the production scale up of polymeric NPs is another challenge and the concentration of produced NPs is generally $<2 \% .{ }^{[24]}$ Lipid nanoparticles is an alternative approach to all-organic nanoparticles through an assembly of small highly lipophilic molecules (lipids). The oldest and actually the most commonly used lipid nanomaterials in nanomedicine are liposomes, which are lipid bilayer vesicles having an aqueous reservoir. ${ }^{[25][26]}$ However, they are not an ideal scaffold for fabrication of stable dye-loaded NPs, because of the lack of organic core. Lipid NPs having organic core can be classified 
based on their physical state: solid lipid NPs (SLNs) and nanoemulsions (NEs). SLNs NPs having a solid lipid core, because they are composed of lipids presenting a solid state at room temperature. ${ }^{[27-29]}$ Their surface is stabilized by non-ionic or ionic surfactants. Although the solid core renders SLNs relatively stable, the fabrication of dye-loaded SLNs is challenging, because the solid lipid can form orientated crystalline domains that gradually expel encapsulated materials to the particle surface. ${ }^{[30]}$ On the other hand, NEs are dispersion of two immiscible liquids, oil in water $(\mathrm{O} / \mathrm{W})$ or water in oil $(\mathrm{W} / \mathrm{O})$ in form of nano-droplets, which are stabilized by an amphiphilic surfactant, with diameter below 300 $\mathrm{nm} .{ }^{[31-33]}$ NEs can be also in form of double ${ }^{[34]}$ and multiple emulsions. ${ }^{[35]}$ Herein, we focus on $\mathrm{O} / \mathrm{W}$ NEs, composed of oil core and stabilized by surfactants forming droplets, dispersed in aqueous continuous phase.

NEs emerged in the past decade as promising delivery platform, due to their long term stability, low toxicity, high encapsulation efficiency, capacity for rapid large scale production and biocompatibility properties. ${ }^{[3,36-40]}$ Importantly, they can be built from components generally recognized as safe (GRAS) and usually FDA approved. ${ }^{[1]}$ They can be formulated easily using nonionic surfactants conferring them a high surface density of PEG, which ensures their stealth properties with respect to immune and rethiculo-endothelial system, ${ }^{[2-45]}$ resulting in the increased circulation time in blood. ${ }^{[46]}$ On the other hand, oil core is a perfect reservoir for solubilizing lipophilic guest molecules at high concentration, in particular, organic dyes to formulate fluorescent NEs.

Another attractive feature of NEs with oily core is that they can be considered as analogues of particles naturally present in the body, such as intracellular lipid droplets, and low-density lipoproteins (LDLs). Lipid droplets are cellular organelles that regulate the storage and metabolism of lipids, which are found in almost every eukaryotic cells, and particularly abundant in adipose tissues. ${ }^{[47,48]}$ Lipid droplets are also a storage for cholesterol and acylglycerols, required for biomembrane formation and maintenance. On the other hand, LDLs are lipid core-protein shell NPs naturally present in blood, and ensure solubilization and transport of lipophilic molecules like cholesterol. LDLs can be considered as a drug nanocarrier, and has already raised interest in nanomedicine. ${ }^{[49,50]}$ Therefore, NEs emerge as bio-mimicking "green" nanocarriers, with strong potential as future safe scaffold for preparation of contrast imaging agents and nanomedicines. In this review, we will focus on NEs as nanocarriers of fluorescent dyes, their fabrication, properties as well as bioimaging and biomedical applications. 
A

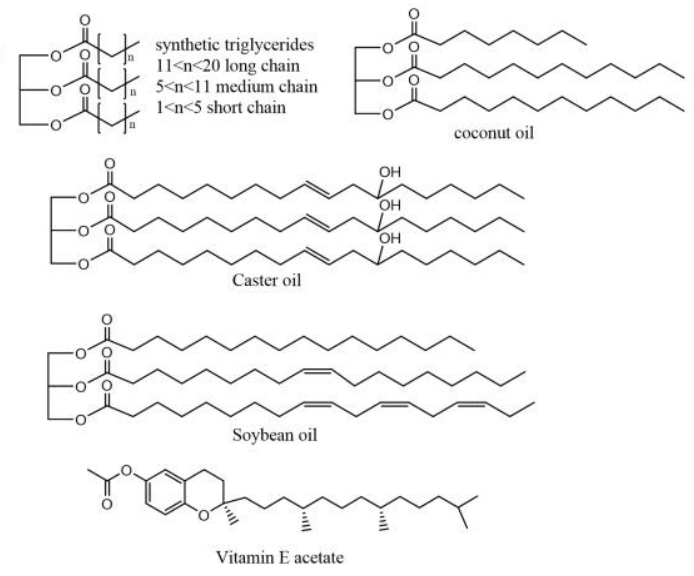

B
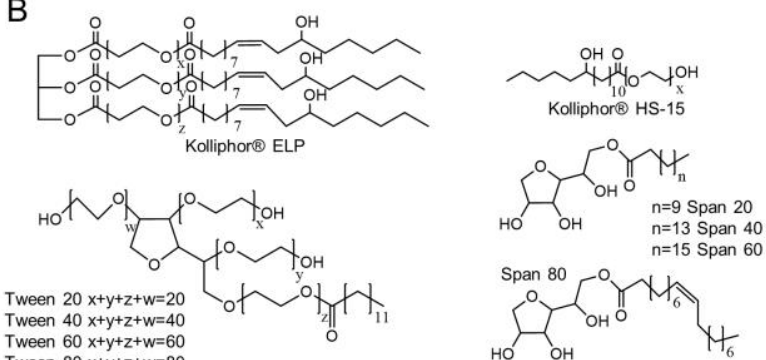

Tween $60 x+y+z+w=60$
Tween $80 x+y+z+w=80$

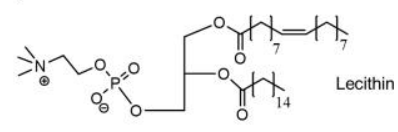

C
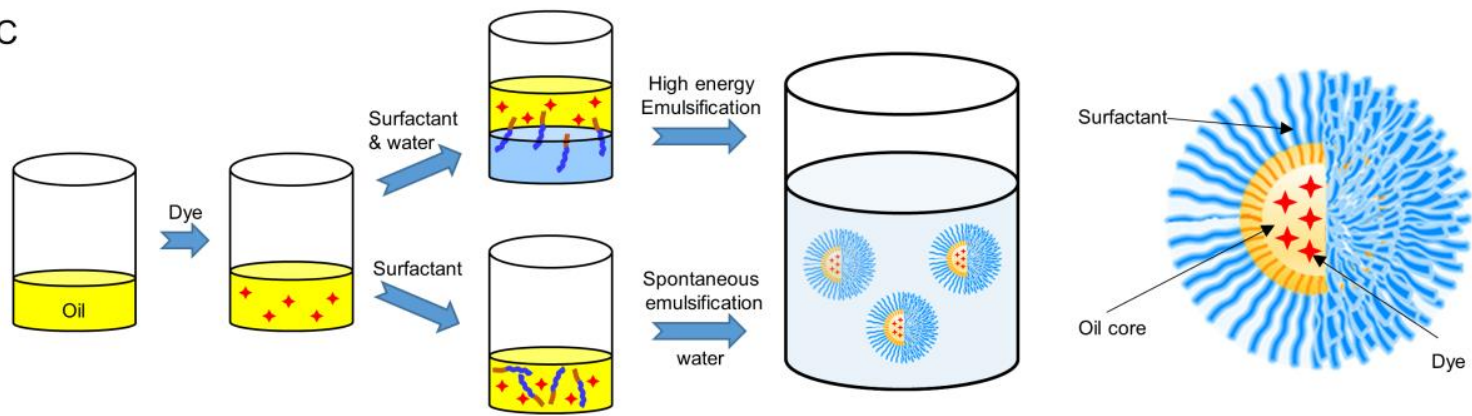

Figure 1. Structure of typical oil (A) and surfactant (B) used in NEs formulation, (C) General procedure for dye loaded NEs formation.

\section{Preparation of dye-loaded NEs}

\subsection{Components of NEs}

\subsubsection{Oil}

A broad range of oils, natural or synthetic can be used in the formulation of NEs. However, biocompatible oil phases from vegetable origin are preferred, such as re-esterified fractions derived from soybean oil, ${ }^{[51-54]}$ sesame oil, ${ }^{[54]}$ cottonseed oil, ${ }^{[55]}$ sunflower oil, ${ }^{[56]}$ coconut oil, ${ }^{[57]}$ rice bran oil (see examples in Figure 1A). ${ }^{[58]}$ A major limitation of natural oils of vegetal origin is their instability. Unsaturated fatty acid esters, present in natural oils (e.g. linseed oil), are susceptible to polymerization reaction in air conditions. ${ }^{[59]}$ Oils based on D- $\alpha$-tocopherol (vitamin E) have also been extensively used in the formulation of NEs. ${ }^{[60-63]}$ Oleic acid and ethyl oleate have been used in oral, topical and parenteral NEs. ${ }^{[64,65]}$ Alternatively, synthetic oils are also widely used, which include long-chain triglycerides (LCT), medium-chain triglycerides (MCT) or short-chain triglycerides (SCT) are used either alone or in combination for formulation of NEs. Among them MCT (e.g. Labrafac $^{\circledR}$ WL) is one of the most common synthetic oils used for formulation of NEs. Solubility of encapsulated compounds (drug or dyes) in the oil phase is an important factor to consider when choosing the oils to be used in NEs formulation 


\subsubsection{Surfactant}

Surfactants are essential for formulation of NEs, because they reduce the interfacial tension and prevent nano-droplets from aggregation. During the formulation process, they rapidly adsorb at oil-water interface of the newly formed droplets, providing stabilization to NEs by steric and/or electrostatic forces. For that purpose, nonionic surfactants are a widely used. They present the advantage to decorate the droplets with a PEG layer along with strongly stabilizing the droplets. Some of them, like ricinoleate PEG-35 amphiphile (e.g. Kolliphor ${ }^{\circledR}$ ELP) are parenteral grade molecules, used in clinical formulations with a variety of oils. ${ }^{[31]}$ Other similar non-ionic surfactants were also employed: Tween 20, 40, 60 and 80 (Polyoxyethylene sorbitan monolaurate), [66-68] Span 20, 40, 60 and 80 (Sorbitan monolaurate) ${ }^{[69,70]}$ and Kolliphor ${ }^{\circledR}$ HS-15 (polyoxyethylene-660-hydroxystearate) (see examples in Figure 1B). ${ }^{[71]}$ In addition, common surfactants of different chemical nature can be also used for NEs preparation: poloxamers (also known as Pluronics), ${ }^{[72]}$ amphiphilic proteins like casein, ${ }^{[73,74]}$ lactoglobulin, ${ }^{[75]}$ polysaccharides (e.g., gums, starch derivatives), ${ }^{[76]}$ and PEG containing block copolymers. ${ }^{[77]}$ Ionic surfactants have also been used for preparation of NEs, like for instance, sodium dodecyl sulfate and docusate sodium salt (AOT). ${ }^{[78,79]}$

Importantly, natural lipids should be also mentioned as emulsifying agents. In particular, phospholipids, like phosphatidylcholine (lecithin, Figure 1B), bearing a zwitterionic head group, ${ }^{[80]}$ and their PEGylated analogues, have been extensively used for preparation of NEs. In case of saturated lipids, their monolayer at the surface was hypothesized to be present in gel (solid) phase, so that these NEs droplets were also called nanocapsules. ${ }^{[81,82]}$ Cholesterol, a natural molecule used for stabilization of liposomes, can also be used for similar purpose in NEs formulations. ${ }^{[83]}$ Ultimately, in a biomimetic approach, lipid membranes from blood cells have also been used to stabilize NEs for in vivo applications. ${ }^{[84]}$

In addition to the formulation of simple oil nano-droplets structures, several literature reports proposed modifications to control their surface properties: for example, the addition of a small amount of amphiphilic polyelectrolytes in the formulation, such as PMAO (poly(maleic anhydride-alt-1-octadecene)), results in the decoration of the droplets interface with carboxylic acid functional groups. ${ }^{[85]}$ An approach based on layer-by-layer (LbL) assembly onto the droplet surface has also been proposed. ${ }^{[86]}$

\subsection{Preparation of NEs: general principles}

Owing to the large interfacial area developed at the water/oil interface of NEs, they are present in a metastable state. Interfacial area indeed involves a high Gibbs free energy of the system according to the expression $\Delta G_{f}=\gamma \Delta A-T \Delta S_{f}$, where $\gamma$ is the interfacial tension, $\Delta A$ - the increase of interfacial area, $T$ - the temperature and $T \Delta S_{f}$ - the entropy of droplet formation. ${ }^{[87]}$ The higher $G_{f}$, the higher the system instability, and this is due to the interfacial energy created $(\gamma \Delta A)$. Therefore, the main challenges in the preparation of emulsions include control of the stability and the need to decrease surface tension as well as the need to supply some energy to increase interfacial area during the formulation. 
In the case of macro-emulsions (diameter higher than micrometer), the stability issue is very important to consider. On the other hand, in the case of nanoemulsions (below $300 \mathrm{~nm}$ ), droplets are so small that, even if $G_{f}$ is much higher, droplets are stabilized because of their size range, that prevents the effect of gravitation (i.e. droplets are Brownian particles that do not cream). In addition, nano-droplets do not flocculate for steric reasons. ${ }^{[88,89]}$ Stability is in fact one of the main interesting features of nano-emulsions compared to other lipid-based nanocarriers.

NEs can be prepared by either high energy or low energy methods. ${ }^{[39]}$ Indeed, the huge interface can be created by supplying a large amount of energy ${ }^{[87]}$ but also by physicochemical (low energy) methods, so-called spontaneous emulsification, ${ }^{[90]}$ with takes benefit of the physicochemical properties of the nonionic surfactants to breaks-up the oil phase into nano-droplets. Spontaneous nano-emulsification method follows very simple processes, where an aqueous phase is added to a homogenized mixture of oil with the surfactants under intensive stirring (Figure 1C). This process is usually done under temperature control, which depends on both oil and surfactant. The simplicity of this formulation method makes it especially attractive, allowing rapid production and industrial scale-up.

\subsection{Layer by layer NEs}

Layer by layer ( $\mathrm{LbL})$ NEs are generally formed by electrostatic interaction of anionic and cationic polyelectrolytes on a surface formed by charged surfactants. In contrast to already described single-layer NEs, the thickness of the hydrophilic shell of NEs can be controlled by varying the number of deposited layers. ${ }^{[91,92]}$ The electrostatic attraction between the oppositely charged species of polyelectrolytes and surfactants leads to polyelectrolyte-surfactant structures that can stabilize the liquid core of NEs. The interfaces of charged surfactant/polyelectrolyte complexes play a crucial role in the formulation of LbL NEs, which include complex formation, competitive adsorption between surface-active polyelectrolyte/surfactant complexes as well as polymer/surfactant aggregates. ${ }^{[1,93]}$ The multilayer structure also depends on non-electrostatic forces, such as hydrophobic interactions and steric repulsion. ${ }^{[94-96]}$

This novel encapsulation process was initiated by Sukhorukov and co-workers ${ }^{[97]}$ with the incorporation of positive and negative polyelectrolytes onto solid colloidal substrates. LbL technology has been successfully applied for preparation of NEs (Figure 2). ${ }^{[98-105]}$ NEs formed by this technique depend on a surfactant-stabilized oil core and LbL shell formed by cationic and anionic polyelectrolytes. Research on LbL NEs is focused on two aspects: novel polyelectrolytes and charged surfactants. Szczepanowicz et al. found out that the major issue in the liquid core $\mathrm{LbL}$ preparation procedure is the instability of polyelectrolyte due to the dynamic nature of micellar aggregates, leading to fast unwanted leakage of encapsulated drug. ${ }^{[92,103]}$ Thus, they developed polyelectrolytes for of stable LbL NEs, which include synthetic (poly(sodium 4-styrenesulfonate) (PSS), poly (diallyldimethylammonium chloride) (PDADMAC), polyallylamine hydrochloride (PAH)), natural (chitosan (CHIT), sodium alginate (ALG)) as well as biodegradable (poly-Lglutamic acid (PGA), poly-L-lysine (PLL)) polyelectrolytes. ${ }^{[103]}$ 
The second direction was dedicated to development of novel ionic surfactants for improving the stability of the oil core. ${ }^{[102-104,106]}$ The construction of stable container shells relies on a successful choice of suitable ionic surfactant for the oppositely charged polyelectrolyte. ${ }^{[104,107,108]}$ For NEs formulation, several ionic surfactants have been developed. Grigoriev et al. ${ }^{[108]}$ prepared a template of ionic surface for the LbL preparation using dodecane nano-droplets stabilized by didodecyldimethylammonium bromide (DODAB). Then, poly-(sodium 4-styrenesulfonate) (PSS)/poly(diallyldimethylammonium chloride) (PDADMAC) polyelectrolytes were used for form multilayers around the droplet core. However, their stability remained low as the oil was released from the nano-capsules to the glass surface. Nilsson and Bergenstahl also reported an DODAB-stabilized NEs of MCT oil core, coated with a hydrophobically modified anionic starch, however this NEs showed aggregation during centrifugation. ${ }^{[109]}$ The latter was attributed to lower electrostatic stabilization of NEs by cationic surfactant DODAB in the presence of the anionic polyelectrolytes. This embarked the search for new surfactant structures and geometric features favoring NEs-templated LbL processes. Sodium dioctyl sulfosuccinate (AOT) was reported to be able to constitute a satisfactory template to encapsulate n-octane nanodroplets in aqueous medium. ${ }^{[103]}$ Bazylli et al proposed a novel type of surfactant, bis-ammonium N,N-bis[3,30-(trimethylammonium)propyl]dodecanamide dimethylsulfate $\left.\left(\mathrm{C}_{12} \text { (TAPAMS }\right)_{2}\right)$ presenting two cationic head groups. It enabled fabrication of LbL coated NEs with improved stability against cargo (dye) leakage. ${ }^{[105]}$

Generally, LbL NEs are highly flexible modular structures and their properties can be readily tuned by the relative concentrations of ionic surfactants and polyelectrolytes, ionic strength and $\mathrm{pH}$ of the medium, or lipophilicity of surfactants. Moreover, change in the $\mathrm{pH}$ can induce disassembly of the NEs and therefore $\mathrm{pH}$-controlled cargo release. ${ }^{[110]}$

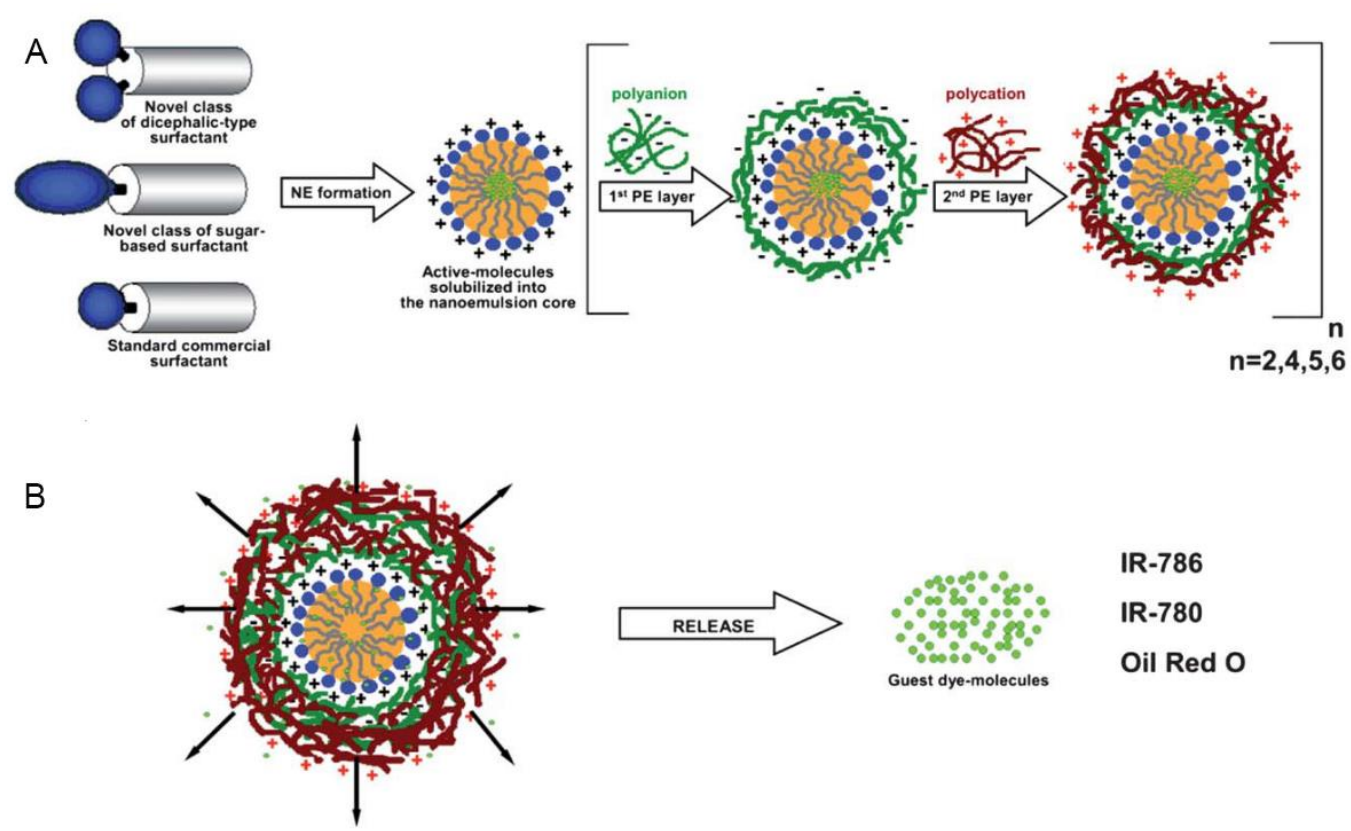

Figure 2. General concept of Bazylli et al of multilayer nanocapsules formation by sequential adsorption of opposite charge polyelectrolyte (PE) layers on the NEs (NE) liquid core: Selected surfactants with different 
hydrophilic head groups used for stabilization of dye-loaded NEs templates covered by " $n$ " PE bilayers; B the release process of studied hydrophobic dye-molecules from the obtained nanocapsule. Reproduced with permission. ${ }^{[105]}$ Copyright 2011 Soft Matter.

\subsection{Dyes and dye-loaded NEs}

Although the dye-loaded nanoparticles have been intensively studied, ${ }^{[3,19,22,23]}$ the research on dye-loaded NEs is still in its infancy. The goal of a successful formulation of dye-loaded NEs is a high and efficient loading, high particle brightness and stability with minimal dye leakage. Indeed, the dye leakage diminishes the brightness of fluorescent NEs, but increases the background because of the leaked dyes. Dyes inside NEs are generally loaded by non-covalent methods, which means that the dye is physically entrapped inside the non-polar oil environment of NEs. In this method, dyes are dissolved in the oil prior to the process of NEs formation. ${ }^{[111]}$ In some cases, the nano-emulsification process needs to be adapted to prevent the dye degradation, for example by modulating the temperature or changing the type of surfactant. ${ }^{[12,113]}$ In cases when dyes have limited solubility in oil, an organic solvent is concomitantly used with dyes, surfactant and oil. ${ }^{[14]}$ It is then evaporated before the nano-emulsification, ${ }^{[115]}$ while the dye solubility in oil is improved due to the amphiphiles (surfactants).

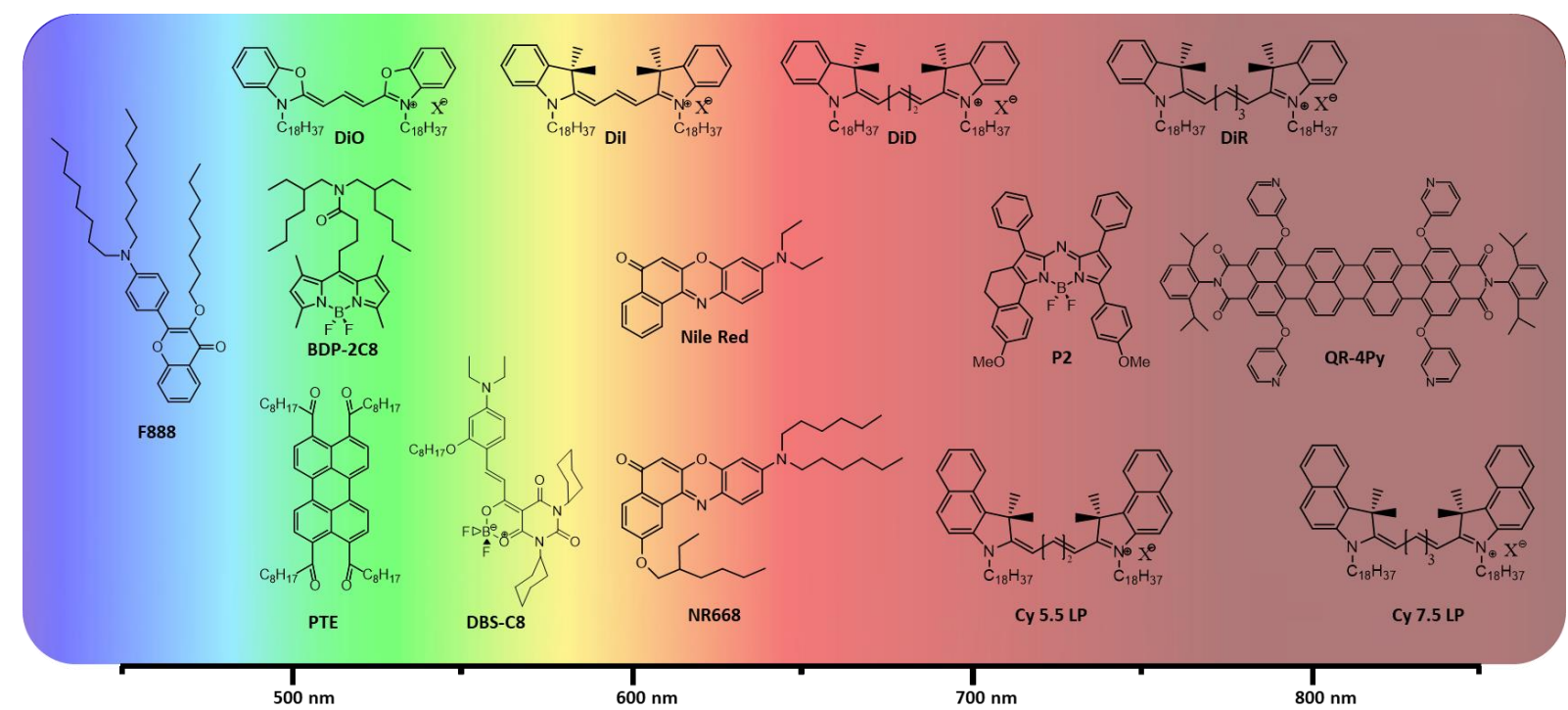

Figure 3. Examples of fluorescent lipophilic dyes used for preparation of dye-loaded lipid NEs organized by their emission wavelength in oil. ( $\mathrm{X}^{-}$is counterion, inorganic anion or tetraphenylborate).

The key to preparation of dye-loaded NEs is proper selection/design of the encapsulated dyes, in order to achieve high solubility in oil, efficient dye loading and high NEs brightness. Variety of dyes have been designed for loading NEs (Figure 3). Several strategies have been followed in the dye design for encapsulation into NEs, which are aimed to improve their lipophilicity. The most common and efficient approach is covalent modification of dyes with lipophilic chains. ${ }^{[42,116]}$ Indeed, practically all examples of dyes for NEs loading bear alkyl chains (Figure 3). The second approach, which is applied to charged dyes (like cyanines) is the use of bulky hydrophobic counterions. ${ }^{[12,113]}$ Increase in 
the dye lipophilicity has several positive effects. First, it improves dye solubility in oil and thus enables efficient loading of dyes and ensures long term stability of NEs without dye precipitation. ${ }^{[42,116]}$ Second, higher lipophilicity will shift the water/oil partition towards the oil core, which inhibit the dye leakage, commonly observed for NEs. ${ }^{[42,112,113]}$ Finally, the dye could be chemically grafted to the components of NEs, such as oil or surfactant molecules. ${ }^{[117]}$ However, this cannot totally solve the problem of dye leakage because the components of NEs are small molecules and can also exchange with biological environment. This and other related issues will be discussed in the next chapter.

\section{Challenges in dye-loaded NEs}

\subsection{Brightness of dye-loaded NEs}

Brightness of dye loaded NEs is crucial for bioimaging applications because it is directly relevant in obtaining images with high resolution. High brightness of dye-loaded NEs is beneficial for the quality of image obtained, because signal-to-noise ratio of the image and the temporal and special resolution of the imaging method are directly linked to the number of capacity of NEs to emit intense fluorescence light. In general, the brightness (B) of a NE droplet is defined by the equation applied to any dye-loaded nanoparticle: ${ }^{[3]}$ $\mathrm{B}=\mathrm{n} \times \varepsilon \times \mathrm{QY}$, where $\mathrm{n}$ is the number of fluorophores loaded in the oily core, $\varepsilon$ and $\mathrm{QY}$ correspond to the extinction coefficient and fluorescence quantum yield of the loaded dye inside NE particle. In the search for brighter dye-loaded NEs, three different ways were considered based on this equation. The first way is to increase the number of loaded dyes, which is represented by the $\mathrm{n}$ in the equation. This can be achieved by firstly increase in the size of NEs, because the larger volume of NEs corresponds to larger $n$ for the same dye concentration. Second is increase in the dye concentration inside NEs. In achieving this purpose, firstly, dye with good solubility in oil is required. As mentioned above, this is usually achieved by modifying dye with lipophilic groups. Long alkyl chains have been used by Texier et al. to improve the optical properties of lipid NEs loaded with cationic cyanine dyes DiD and DiR. ${ }^{[14]}$ Both of them were encapsulated into NEs with encapsulation efficiency of $99 \%$ to reach dye concentration of $3.9 \mathrm{mM}$ in oil core, without any observed fluorescence self-quenching. An increase in the fluorescence quantum yield $\Phi$ from 0.28 to 0.38 and fluorescence lifetimes $\tau$ from 1.0 to $1.8 \mathrm{~ns}$ were observed for DiD (Figure 3 ) inside NEs vs. organic solvent. Similar tendency was observed for DiR (Figure 3): $\Phi$ increased from 0.13 to 0.25 , while $\tau$ increased from 0.57 to $1.1 \mathrm{~ns}$. These effects were assigned to more viscous environment of the oil core compared to the organic solvent. Having 350 dyes per particle, the brightness of DiD-loaded particles scored up to $1.6 \times 10^{7} \mathrm{M}^{-1} \mathrm{~cm}^{-1}$, ${ }^{[118]}$ this brightness are close to the reported highest values for QDs $\left(\sim 4 \times 10^{6} \mathrm{M}^{-1} \mathrm{~cm}^{-1}\right),{ }^{[119]}$ dyeloaded silica NPs $\left(\sim 2 \times 10^{6} \mathrm{M}^{-1} \mathrm{~cm}^{-1}\right),{ }^{[120]}$ and polymeric micelles $\left(\sim 5 \times 10^{5} \mathrm{M}^{-1} \mathrm{~cm}^{-1}\right) .{ }^{[121]}$

When dyes are loaded at high concentration, a fundamental problem of aggregation caused quenching (ACQ) arise because of the close proximity of the encapsulated fluorophores. ${ }^{[3]}$ In our previous studies, we showed that Nile Red and NR688, self-quench at high concentrations inside the oil core, indicating that its planar structure favors dye 
aggregation at high concentrations. ${ }^{[42]}$ Dye aggregation can also result in the destabilization of NEs and sometimes in dye precipitation. ${ }^{[42]}$ Therefore, ACQ should be avoided as much as possible. There are several ways to prevent ACQ behavior in dye-loaded NPs ${ }^{[3]}$ : (i) aggregations-induced emission; (ii) introduction of bulky groups; and (iii) bulky hydrophobic counterions for ionic dyes. AIE is one of the most popular methods for fabrication of organic materials emissive in the solid state. ${ }^{[122]}$ However, so far, AIE dyes (AIEgens) have not been directly used to prepare fluorescent NEs. On the other hand, they have been successfully used to stain lipid droplets inside the cells, ${ }^{[22,123,124]}$ which are nanoobjects of similar structure. Incorporation of bulky groups can provide direct steric hindrance to $\pi$-stacking, which inhibits $\mathrm{H}$-aggregation. This approach was particularly used in solutions, ${ }^{[125,126]}$ solid state ${ }^{[127]}$ and in dye-loaded polymeric materials. ${ }^{[128]}$ It is also attractive for dye-loaded NEs, because incorporation of bulky lipophilic groups can both improve oil solubility and decrease the ACQ. For example, 3-alkoxychromone derivative F888 (Figure 3), which bears bulky groups to prevent direct $\pi-\pi$ stacking showed a remarkable resistance to ACQ even at very high dye loading, showing up to $78 \%$ fluorescence QY at $10 \mathrm{wt} \%$ loading in the oil core. ${ }^{[42]}$

In case of DiD and DiR bearing long alkyl chains used for loading into NEs, ${ }^{[14]}$ high loadings were not studied, probably because of problem of dye solubility and aggregation. The point is that cationic dyes, such as cyanines, are prone to form selfquenched $\pi$-stacked structures (H-aggregates) at high concentration, ${ }^{[129]}$ which are responsible for strong ACQ. We recently found that ACQ in charged dyes in dye-loaded polymer and lipid NPs can be prevented by replacing their small hydrophilic counterion (i.e. perchlorate, iodide, etc) with bulky hydrophobic counterions. ${ }^{[12,130]}$ The ion pair of cationic cyanine dye Dil with tetraphenylborate TPB (DiI-TPB, Figure 4), showed $>40$-fold improved solubility in labrafac, allowing dye loading up to $8 \mathrm{wt} \%{ }^{[112]}$ It was remarkable that even at $8 \mathrm{wt} \%$ DiI-TPB loading only negligible dye aggregation was observed judging from the optical absorption measurements of the dye-loaded NEs. Only 3.5-fold decrease in NEs quantum yield was observed by increasing the dye concentration by 80 -fold (from 0.1 to $8 \mathrm{wt} \%$ ). This led to the increase of estimated particle brightness 23-fold (80/3.5) compared to the NEs with of $0.1 \%$ dye loading (Figure 4B). The obtained $90 \mathrm{~nm}$ NEs encapsulating $~ 12000$ dyes per droplet showed a quantum yield of 0.14 , which corresponded to theoretical brightness $\mathrm{B}=2.5 \times 10^{8} \mathrm{M}^{-1} \mathrm{~cm}^{-1}$. This value was close to that obtained experimentally $\left(\mathrm{B}=8.0 \times 10^{7} \mathrm{M}^{-1} \mathrm{~cm}^{-1}\right)$ by single-particle microscopy with reference particles (FluoSpheres ${ }^{\mathrm{TM}}$ ) of known brightness (Figure 4C). The latter value was $>100$-fold brighter than quantum dots of similar emission wavelength with quantum yield of $\sim 0.5$ and extinction coefficient at the long-wavelength maximum of $\sim 1 \times 10^{6}\left(\mathrm{~B} \sim 5.0 \times 10^{5} \mathrm{M}^{-1}\right.$ $\left.\mathrm{cm}^{-1}\right)$. 

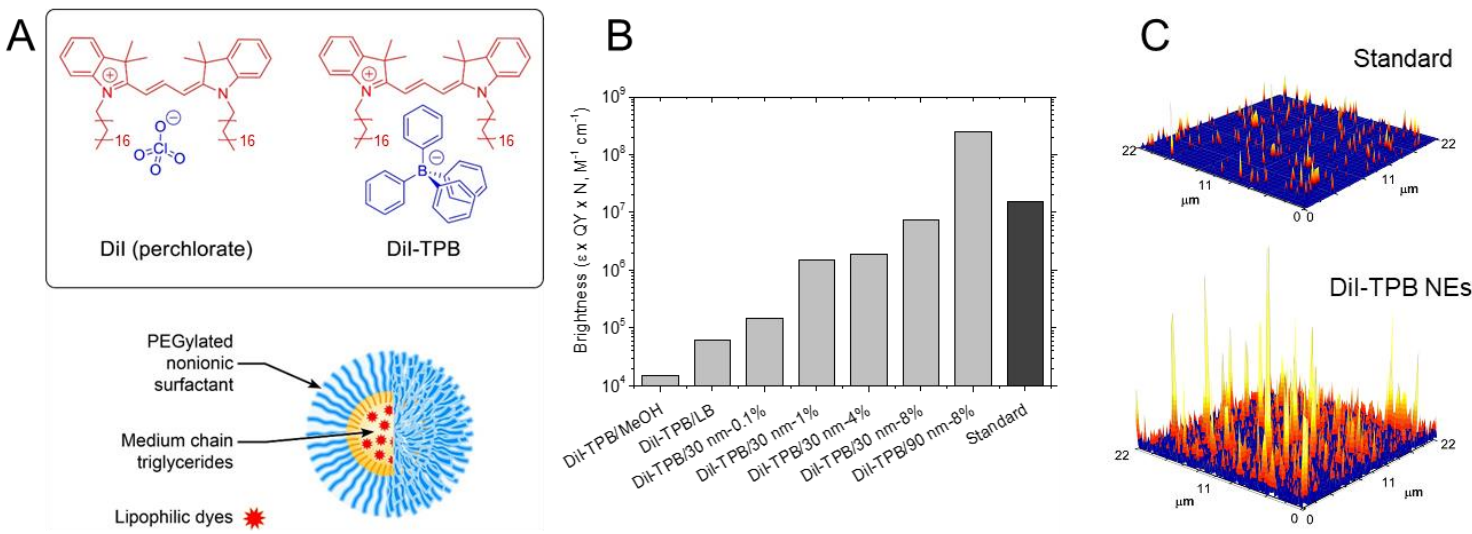

Figure 4. Chemical structures of DiI perchlorate and DiI-TPB and schematic presentation of a nano-droplet encapsulating them. With confocal images of single nano-droplets loaded with DiI-TPB at $8 \mathrm{wt} \%$ (A) and standard polystyrene fluorescent nanoparticles (FluoSpheres ${ }^{\mathrm{TM}}$, Red-orange, $565 / 580 \mathrm{~nm}, 45 \mathrm{~nm}$ diameter) of similar spectroscopic properties (B) in agarose gel recorded at the same experimental conditions. Reproduced with permission. ${ }^{[112]}$ Copyright 2014 Biomaterials.

Brightness of dye-loaded NEs is also closely associated with the characteristics of dye itself, notably its extinction coefficient and quantum yield. A lot of research have been done in the past few years on the developing of novel dyes with improved brightness characteristics, ${ }^{[131]}$ although the efforts to design bright dyes specially for NEs are still limited. Earlier, Zyabrev et al. ${ }^{[132]}$ introduced the dioxaborine barbituryl moiety and then Kovtun et al designed new fluorophores based on dioxaborine complexes and indolenine moieties. ${ }^{[133-135]}$ By combining these design approaches, we recently reported a series of fluorophores called StatoMerocyanines. They are composed of an indolenine moiety and a bulky barbiturate rigidified by a dioxaborine complex, which are ultrabright in oil and used as lipid droplet markers in cellular and tissue imaging. ${ }^{[136]}$ They displayed remarkable high molar extinction coefficients (up to $390000 \mathrm{M}^{-1} \mathrm{~cm}^{-1}$ ) and quantum yields up to $100 \%$. However, the dye compatible with common $488 \mathrm{~nm}$ excitation (SMCy3) showed modest quantum yield of 0.25 and extinction coefficient of $87,300 \mathrm{M}^{-1} \mathrm{~cm}^{-1}$. Therefore, the indolenine group was replaced with aniline unit yielding new fluorophores called dioxaborine barbituryl styryl dyes (DBS) and incorporating long alkyl chain (DBS-C Figure 3). This dye showed a good molar extinction coefficient of $120000 \mathrm{M}^{-1} \mathrm{~cm}^{-1}$ (two times higher than Nile Red) and an increased quantum yield of 0.98 (similar to Nile Red). ${ }^{63]}$ The brightness of DBS fluorophores in oil was enhanced due to their viscosity, ${ }^{[63]}$ a phenomenon similar to that observed earlier by Texier et al using cyanines. ${ }^{[14]}$ The developed DBS dyes showed highest brightness (quantum yield and molar extinction coefficient) in castor oil (with the highest viscosity) compared with other oils (e.g. MCT and VEA). The fluorescence quantum yield correlated with solvent viscosity, so that it was higher in oils of higher viscosity (Figure 5). It can be explained by the restriction of intramolecular rotation, commonly observed in fluorophores with structure of molecular rotors. ${ }^{[137,138]}$ Consequently, it is recommended to use viscous oil to increase the brightness of NEs when loading fluorophores with high degree of rotation freedom. On the other hand, 
dyes with rigid structure, like NR668, do not show a clear correlation of their fluorescence quantum yield with the oil viscosity (Figure 5).

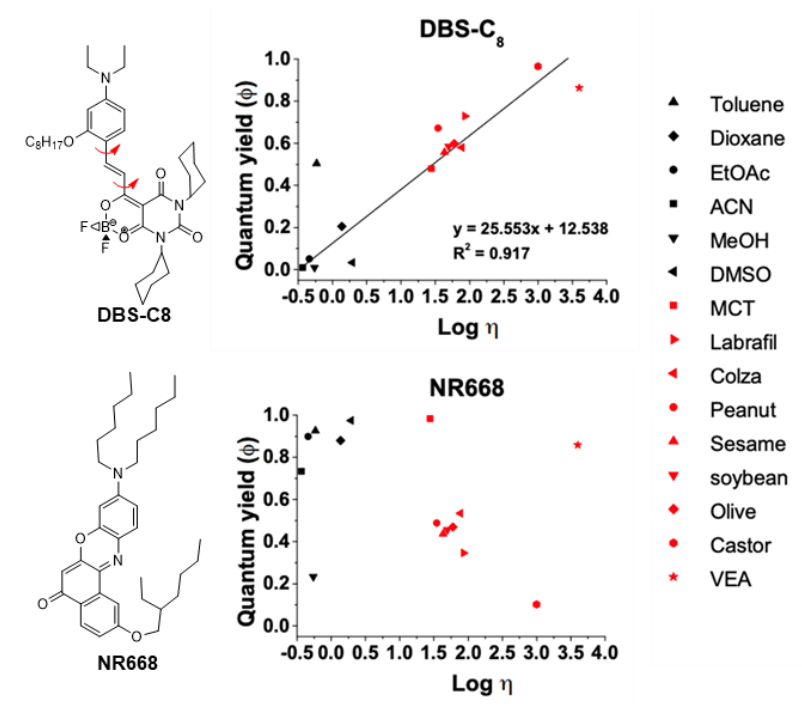

Figure 5. Correlation of the fluorescence quantum yield of DBS dyes and NR668 with the viscosity of the medium. $\eta$ is the solvent viscosity expressed in mPa.s. Red arrows show rotational freedom in DBS- $\mathrm{C}_{8}$ dye, responsible to viscosity effect. Adapted with permission. ${ }^{[63]}$ Copyright 2019 ACS Appl. Mater. Interfaces.

\subsection{Photostability and phototoxicity}

High photostability of dye-loaded NEs is almost as important as brightness for highquality fluorescence imaging, because it defines the number of photons emitted by the system before complete photobleaching. Photobleaching, which is a loss of fluorescence intensity (brightness) over time on continuous exposure to light, is a common problem of organic dyes. It is frequently observed in cellular microscopy experiments when the same region of cells is repeatedly imaged. In the study of Gravier et al, ${ }^{[118]}$ the increase in the DiD concentration from 1.2 to $4 \mathrm{mM}$, increased in the photobleaching rate by $63 \%$. However, further increasing the concentration to $10 \mathrm{mM}$ decreased the photobleaching rate to the same as $1.2 \mathrm{mM}$. The first phenomenon was attributed to photoproduced oxidized radicals that could react with neighboring fluorophore. By increasing the dye concentration, the bleaching reaction should be faster when the dye concentration is below dye self-quenching concentration. However, after the dye loading concentration reached the self-quenching value, dye would form aggregates, thus lower down the concentration of reactive dye species concentration, leads to a higher resistance to photobleaching. In another study, a photostability in dye-loaded NEs was achieved by using fluorinated oil core encapsulating specially designed NIR quaterrylene dye (QR-4Py, Figure 3). ${ }^{[139]}$ In high polar medium, fluorescence of this dye was quenched, because the highly rigid and planar aromatic structure favored the $\pi-\pi$ stacking in aqueous solutions. Inside NEs it drastically increased its emission and showed much higher photostability compared to corresponding formulation with DiR dye.

The encapsulation of dyes in a LbL NEs system could help to increase the photostability and, at the same time, increase the generation of ROS species. It was reported 
that the encapsulation of a cyanine dye IR-786 in multilayer oil core nanocapsules increased the dye photostability and conserved its photodynamic effectiveness. This photostability effect of LbL NEs was proved by a model study, where a higher ability to produce ROS was observed for encapsulated IR-786 in comparing to the free form. ${ }^{[140]}$ Photostability is also closely linked to phototoxicity, because both are related to formation of the dye triplet state and further generation of highly reactive singlet oxygen (see a dedicated chapter below). On one hand, this would lead to unfavorable phototoxic effects, especially at strong irradiance. On the other hand, phototoxicity of NEs can be directly used in photodynamic therapy $(\mathrm{PDT})^{[141]}$ as generated singlet oxygen can kill target pathological cells (see a dedicated chapter below).

In addition to photo-degradation of encapsulated dyes, intense light irradiation can directly affect the stability of dye-loaded NEs. ${ }^{[142]}$ Indeed, our earlier studies with NEs loaded with NR688 dye showed that strong irradiation for a few seconds, can induce, in addition to photo-degradation of the dye, its effective release into biological environment. The effect was found to be oxygen-dependent, indicating that that some photochemical degradation of the NEs and/or dye could trigger dye release of NEs. This originally unwanted phenomenon was then applied for a light-controlled release of NEs cargos in cells and in zebrafish embryo. ${ }^{[142]}$

\subsection{Stability of dye-loaded NEs and cargo release}

When the dyes are non-covalently encapsulated inside a nanocarrier, they may undergo a leakage that depends on both dye and nanoparticle platform. ${ }^{[143]}$ As mentioned above, it is important to prevent the dye leakage, because it decreases the particle brightness, but increases the background signal caused by the released dyes. Therefore, the leakage lowers the imaging contrast obtained with the dye-loaded NEs and can be a source of important artifacts. ${ }^{[42]}$ In contrast to dye-loaded nanoparticles based on silica ${ }^{[144]}$ and polymers, ${ }^{[145]}$ which are generally more resistant against dye leakage due to their solid core, nano-droplets with their liquid core are more prone to the leakage. As a result, they usually release their lipid content rather rapidly ranging from minutes to hours depending on the lipophilicity and the structure of the encapsulated molecule. ${ }^{[146,147]}$ This might be the reason why fluorescent NEs are less common imaging agents than solid NPs. The retention of a dye in NEs depends strongly on hydrophobic interactions between the dye and NEs. Therefore, highly hydrophobic dyes will likely have a strong preference for the oil phase, thus limiting its leakage in biological environment. Moreover, more lipophilic dyes will remain in the oil core and less at the NEs-interface, which slow down exchange with continuous phases and acceptor-media like biological environment (blood, other biological fluids, tissues) ${ }^{[42]}$ In this case, the dyes are released only when the NEs degrade releasing its oil, which is optimal for bioimaging and cargo delivery applications. An additional stability can be provided using fluorinated emulsions based on fluorinated oil, which provides NEs core with superhydrophobic properties. It was shown that dyes bearing fluorinated alky chains exhibit much higher stability against dye leakage inside fluorinated emissions than their non-fluorinated 
analogues ${ }^{[148]}$ highlighting the importance of chemical match between the dye cargo and the oil core.

However, characterization of dye release from hydrophobic reservoir of NEs and NPs is challenging using classical methods, such as dialysis. ${ }^{[149,150]}$ The point is that hydrophobic cargo is poorly soluble in water, so that in the conditions of dialysis in pure water, NEs would produce false impression of good stability. Unfortunately, the use of acceptor media containing organic solvents (like ethanol and isopropanol) is not compatible with NEs because they can destabilize the nano-droplets. Moreover, dialysis is difficult to realize in the presence of serum, as serum particles are much larger than dialysis membrane and could alter its function. Analysis of particle size is very useful to understand stability, although dye leakage is not always associated with changes in the particle size and measurements of particle size in complex biological systems is prone to artifacts.

Methods exploiting light absorption and fluorescence of the encapsulated dyes can help to understand the problem of dye leakage and NEs stability. The simplest approach uses optical spectroscopy, where the dye aggregation or leakage can lead to changes in the absorption and emission wavelengths. ${ }^{[151-154]}$ One of the most powerful methods to understand particle integrity is Forster resonance energy transfer (FRET). Since short donoracceptor distances are required for the efficient FRET $(2-10 \mathrm{~nm})$, one should achieve $\mathrm{mM}$ concentrations of dyes to realize FRET inside the nano-droplets. This means that the efficiency of FRET can be used as the tool to monitor the concentration of dyes loaded in the NEs and thus dye leakage behavior. This approach was successfully used for variety of NPs in order to understand their stability and the cargo leakage in solution and directly in small animals: polymeric NPs, ${ }^{[155]}$ conjugates with quantum dots, ${ }^{[156]}$ and dye-loaded lipid NPs. ${ }^{[157]}$ When using this technique, the FRET pair should be carefully chosen in order to have good overlap between emission band of the donor and the absorption band of the acceptor, while keeping donor and acceptor emission bands well separated to avoid their overlap. ${ }^{[158,159]}$ A complementary approach to study stability of fluorescent NPs is Fluorescence correlation spectroscopy (FCS), a well-known technique in biophysics ${ }^{[160]}$ and materials science ${ }^{[161]}$ that allows to study in situ diffusion of emissive species. Similar to DLS, it can provide information about the hydrodynamic radius, but it can in addition be used in situ in biological media, including serum, which is challenging for DLS. Therefore, it was successfully used to study interactions of NPs with serum proteins and formation of protein corona of NPs. ${ }^{[3,162]}$ The unique feature of FCS is that it can provide information about brightness and the number of emissive species. The two parameters are directly linked to the stability of NPs, because once the fluorescent cargo is released into recipient medium (e.g. serum), the number of emissive species increases while their brightness decreases. ${ }^{[42,150]}$

In our early studies, we focused on the development of stable non-leaking dye-loaded NEs based on Nile Red. ${ }^{[42]}$ To ensure proper loading and stability against dye leakage, parent Nile Red was functionalized with three hydrophobic chains (NR668, Figure 3). Then, using FRET and FCS techniques, we compared stability of NEs loaded with NR668 vs the parent analogue: Nile Red. To design FRET NEs, they were loaded with a FRET donor dye 
F888 (Figure 3), which is blue dye also bearing three lipophilic chains and its emission showed good spectral overlap with the absorption of Nile Red dyes. Efficient FRET could be achieved at 0.5 wt.\% loading of both donor (F888) and acceptor (Nile Red or NR668) (Figure 6A,B). Then incubation in serum revealed that FRET NEs based on Nile Red acceptor lost their FRET signal already after $3 \mathrm{~min}$ of incubation, whereas only minor release was observed for NEs loaded with NR668 after $6 \mathrm{~h}$ at $37{ }^{\circ} \mathrm{C}$ (Figure 6C). This result indicated that Nile Red is not hydrophobic enough to remain in the oily core of nanodroplets and thus undergoes a rapid leakage in biological media. On the other hand, the lipophilic version of Nile Red, NR668, shows excellent stability against leakage. The stability of NEs was then studied by FCS through monitoring the brightness and number of the emissive species in the serum medium. ${ }^{[42]}$ In this case, Nile Red-loaded NEs showed rapid increase in the number of emissive species and drop of the particle brightness already after $5 \mathrm{~min}$ of incubation in serum, whereas these parameters were relatively stable for NR668 cargo. These FCS results correlated well the FRET data, confirming the importance of lipophilic alkyl chains in the stability of NEs. Finally, NEs loaded with Nile Red showed rapid leakage in presence of cells, whereas NR668-loaded NEs were resistant for hours in the same conditions. ${ }^{[42]}$ Later studies using Nile Red and NR668 showed that lipophilicity of cargo is crucial for controlling exchanges and interactions between loaded and empty NEs as well as between NEs and live cells. ${ }^{[163]}$ In addition, using both FRET and FCS we found that NEs encapsulating NR688 can be destabilized only after reaching high temperature, such as $60{ }^{\circ} \mathrm{C}^{[150]}$ Moreover, this study showed that by measuring the standard deviation of fluorescence fluctuation in FCS, one can provide a quantitative measure of NEs cargo release in situ in biological medium.
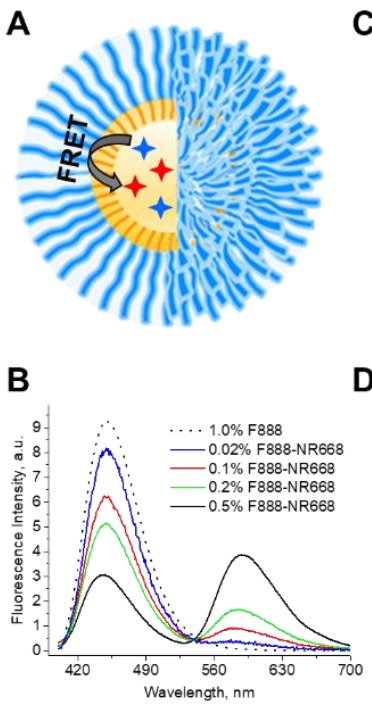
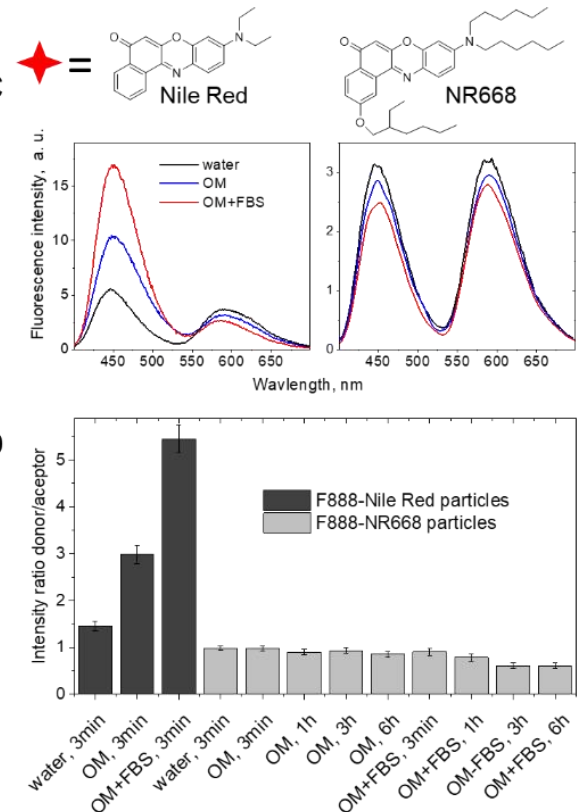

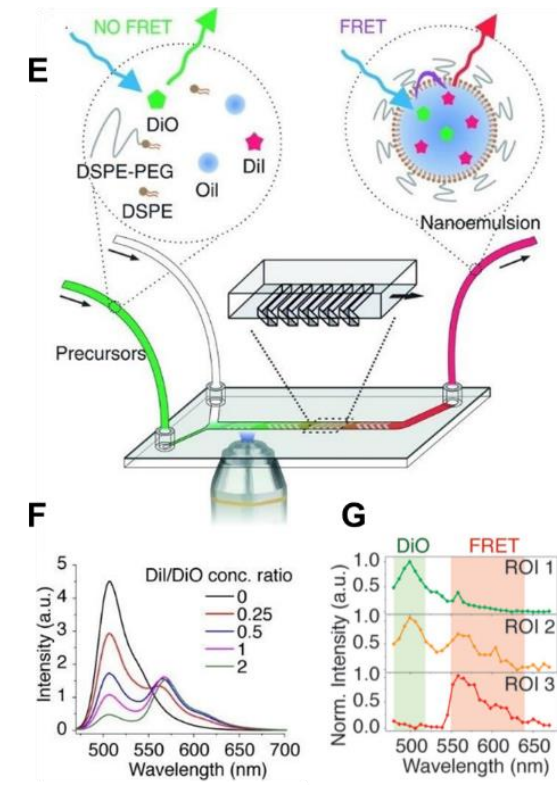

Figure 6. (A) Schematic presentation of FRET NEs. (B) Fluorescence spectra of FRET NEs at different concentrations of donor (F888) and acceptor (NR668). The black dotted line corresponds to NEs containing only the donor (1\% loading). Fluorescence spectra were recorded at $390 \mathrm{~nm}$ excitation wavelength. (C) Investigation by FRET of the dye release from NEs in different media: water, Opti-MEM (OM) and OptiMEM with 10 vol.\% FBS (OM+FBS). NEs encapsulating $0.5 \mathrm{wt} \%$ of F888 as energy donor and $0.5 \mathrm{wt} \%$ of Nile Red or NR668 as energy acceptor were used. The NEs were diluted 10 000-times from the original 
formulation into the medium of interest. The first fluorescence spectra were measured after 3 min incubation at RT. Then, samples were incubated for $1 \mathrm{~h}, 3 \mathrm{~h}$ and $6 \mathrm{~h}$ at $37{ }^{\circ} \mathrm{C}$. (D) FRET was quantified as the fluorescence intensity ratio between the maximum of the donor $(450 \mathrm{~nm})$ and acceptor $(590 \mathrm{~nm})$. The donor in the nanodroplets was excited at $390 \mathrm{~nm}$. (E) Schematic illustration of the microfluidic setup to study formation of NEs by FRET. (F) Emission spectra of NEs containing different DiI/DiO concentration ratios, excited at $458 \mathrm{~nm}$. (G) Emission spectra recorded inside microfluidic device at indicated ROIs: ROI 1 corresponds to the organic phase; ROI 2 lies at the periphery of the solutions mixing area, and the ROI 3 corresponds to the central mixing area, where NEs are expected to form. (B-C) Adapted with permission. ${ }^{[42]}$ Copyright 2012 RSC Adv. (E-G) Adapted with permission. ${ }^{[164]}$ Copyright 2017 Angew. Chem. Int. Ed.

The stability of dye-loaded NEs in plasma was also studied using FRET between cyanine dyes. ${ }^{[165]}$ NEs loaded with FRET donor (DiD) and acceptor (DiR) showed slow decrease in the FRET efficiency starting from $1 \mathrm{~h}$ and the process ended after $24 \mathrm{~h}$, indicating good resistance of NEs to leakage. Owing to NIR emission of the DiR acceptor, these FRET NEs allowed studying the biodistribution and stealth properties of NEs in mice. However, FRET NEs in this case showed low relative intensity of acceptor, indicating low FRET efficiency. The reason is probably linked to low solubility of positively charged cyanines dyes, DiD and DiR in oil, which does not allow achieving sufficiently high concentration of cyanines inside NEs. Solubility and loading of cationic cyanines can be drastically improved (> 80-fold) by simply replacing their hydrophilic inorganic counterion with bulky hydrophobic counterion (e.g. tetraphenylborates, TPB), which make cyanines much more lipophilic. ${ }^{[112]}$ Following this finding, we were able to prepare NEs with highly efficient FRET by loading two NIR dyes inside NEs at sufficiently high concentration $(1 \mathrm{wt} \%)$. ${ }^{[13]}$ Cyanines Cy5.5 LP and Cy7.5 LP, bearing octadecyl hydrophobic chains and TPB counterions were used for encapsulation. Remarkably, these NEs showed practically no loss of FRET signal after $24 \mathrm{~h}$ incubation in serum. This higher stability is probably related to the presence of bulky counterion in cyanines, which make them less prone to leakage. Moreover, having both donor and acceptor emitting in NIR region, we were able to perform quantitative analysis of FRET of NEs injected in mice. Experiments on both healthy and tumor bearing mice revealed remarkable stability of NEs in blood circulation (see below). Later on, Roger et al. employed another pair of cyanine dyes, DiI and DiD, with TPB counterion to study integrity of NEs while crossing the intestinal barrier. ${ }^{[166]}$ It was shown that FRET signal of NEs was preserved after their transfer across an intestinal epithelium model, indicating the capacity of NEs to undergo transcellular crossing without losing their integrity.

Another interesting application of FRET with NEs was to monitor their formation process. Sanchez Gaytan et al. ${ }^{[164]}$ used a micro-flow-homogenizer to mix basic components to form NEs along with two lipophilic dyes, DiO and DiI, upon NEs formulation, the FRET pair was encapsulated into the oil core, triggering FRET (Figure 6E). As the NEs formed, an increase in the acceptor fluorescence intensity and decrease in the donor fluorescence intensity were observed, so that the color changed from the green to red (Figure $6 \mathrm{~F}, \mathrm{G}$ ).

Recently, we exploited two other methodologies to generate fluorescence response to dye leakage. In one study, we used the phenomenon of self-quenching of NR668, which is 
observed at high dye loading inside NEs. Then, during dye transfer to a recipient medium (e.g. empty NEs), dilution of the dye led to de-quenching, i.e. to increase in the fluorescence intensity, which allowed monitoring the dye release. ${ }^{[167]}$ In a second approach, we designed a fluorogenic dye, which light up on hydrolysis of its ester bond after release from the nanocarrier. ${ }^{[168]}$ This approach is especially suitable for monitoring intracellular release of model lipophilic pro-drugs followed by activation of active cargo by intracellular esterases.

To minimize dye leakage, it is also possible to directly graft the dyes to the oil or surfactant component used for the formation of NEs. ${ }^{[169]}$ Patel et al. described an approach to monitor a two oil phase NEs system where each component was separately labeled by fluorescent dyes, namely, Cy3 was connected with perfluoropolyether (PEPE) oil and DiR dissolved in the outer hydrocarbon oil phase. This formulation showed great size stability and resistance to fluorescence signal losing over time. After 49 days stored at $4{ }^{\circ} \mathrm{C}$, fluorescence signals of $\mathrm{Cy} 3$ dye barely decreased (4\%), while DiR on the outer layer showed a reduced fluorescence of $23 \%$. Jarzyna described a way to formulate a multifunctional nanoparticle system with Cy5.5 grafted on the lipid surfactant (DSPE-PEG) at its PEG end and iron oxide encapsulated in the oil core. ${ }^{[115]}$ This method required the pre-synthesis of the surfactant part, which is called Cy5.5-PEG-DSPE. The reduced dye leakage in the obtained NEs allowed non-targeted imaging of subcutaneous tumors.

Another way to prevent leakage of dyes is by using cationic lipid that provide ionic interactions inside oil core, similar to bulky hydrophobic counterion TPB, mentioned above. It is reported that cationic lipid stearylamine (SA) can enhance stability of NEs loaded with indocyanine green (ICG), a water-soluble, amphiphilic anionic tricarbocyanine dye. The incorporation of this lipid would trigger electrostatic interaction with nearby anionic ICG, thus preventing ICG from aggregation and destabilization of the NEs. ${ }^{[170]}$

The multi-layer NEs system could also be used to prolong the release of dyes, when the $\mathrm{C}_{12}$ (TAPAMS $)_{2}$ templates were coated with 2, 4, 5 and 6 layers of PSS/PDADMAC polyelectrolyte bilayers, the release of encapsulated cyanine dyes was slowed by 5, 30, 340 and 13400 times, respectively, compared to the bare NEs. ${ }^{[151]}$ The release rate depends on the interfacial tension and surface activity in this case and can be modified by increasing the number of layers in the polyelectrolyte coating.

Further control on the encapsulation and release of cargo from NEs was achieved by embedding NEs into hydrogels. In this case, NEs were photo-polymerized inside poly(ethylene glycol) diacrylate and loaded with lipophilic cyanine dyes (PKH26, DiI analogue) and $\mathrm{DiO}$ as well as model cargo, Nile Red and fluorescently labelled protein streptavidin. ${ }^{[171]}$ Prolonged $\mathrm{pH}$-controlled release of fluorescent cargos was demonstrated due to degradation (cleavage of acrylic esters) of the hydrogel matrix.

\subsection{Surface modification of NEs}

Surface functionalization of nanocarriers is a key step towards biomedical applications that include targeted imaging and drug delivery. However, so far, surface modification of NEs remains challenging because of their liquid nature. Indeed, in contrast to solid inorganic and 
organic NPs, the surface of NEs is highly dynamic because surfactant can readily exchange with the environment and thus loose a grafted functionality. Nevertheless, a number of approaches for NEs functionalization have already been proposed.

The most common one is based on functionalization of PEGylated lipids (e.g. DSPE-PEG), which insert at the NEs interface as a surfactant. On the one hand, it allows grafting fluorescent dyes to the NEs surface (see above). ${ }^{[15]}$ On another hand, DSPE-PEG enables grafting tumor targeting ligands, like RGD. In the study by Texier and co-workers, $9.2 \%$ of surfactant was replaced with DSPE-POE5000-maleimide, which bears thiol reactive group used for grafting RGD moiety. ${ }^{[172]}$ PEGylated surfactant bearing stearate lipophilic chain and reactive groups have also been used for chemical functionalization with RGD ligand. ${ }^{[173]}$ More recently, Niko and co-workers proposed an original approach, where the commonly used surfactant for formulation of NEs, Kolliphor ${ }^{\circledR}$ ELP (Cremophor ${ }^{\circledR}$ ELP, Figure 1B), was functionalized with azide groups. ${ }^{[174]}$ These obtained azide-bearing NEs were successfully functionalized with cell penetrating peptides, which was applied to enhance their internalization into cancer cells in vitro. In another recent study, lipophilic amine oleylamine was used to graft targeting ligand RGD and fluorescein to NEs, which showed higher cellular update in vitro compared to analogues with RGD ligand. ${ }^{[175]}$

An emerging strategy to modify NEs surface is to use amphiphilic polymers. In a recent study, NEs were formulated with the lipophilic poly(maleic anhydride-alt-1-octadecene) (PMAO), which can hydrolyze into a amphiphilic polymer containing negatively charged carboxylates localizing at the NEs-water interface. ${ }^{[176]}$ The attractive feature of this approach is that amphiphilic polymer, bearing multiple lipophilic tails should provide more stable surface modification, in comparison to methods based on amphiphiles bearing 1-2 lipophilic tails. Further studies showed that PEGylated PMAO derivatives can be used to prepare fluorescent hybrid lipid-polymer NPs, where the lipid droplets, loaded with lipophilic BODIPY dye BDP-2C 8 (Figure 3), are decorated with stable a PEG shell. ${ }^{[177]}$ PEG shell at the NEs surface was also introduced using a radical polymerization of poly(ethylene glycol)diacrylate, and the oil core-PEG shell NEs were successfully loaded with lipophilic contrast agents, such as iron oxide nanocubes and fluorescent dye indocyanine green. ${ }^{[178]}$

\section{Biological applications of fluorescent NEs}

\subsection{Imaging at the cellular level}

\subsubsection{Studying the pathway of NEs entering cells}

Dye-loaded NEs showed the potential to be used to understand the interactions of these nanocarriers with cells. Particularly important is the internalization of NEs and the release of their content to the cells. Generally, there exist two main paths for the internalization of NPs: endocytosis ${ }^{[179]}$ and macropinocytosis. ${ }^{[180]}$ It was shown that NPs with size above $300 \mathrm{~nm}$ preferentially internalize through macropinocytosis, whereas below this size they usually undergo endocytosis. ${ }^{[181]}$ As the size range of NEs is usually between 20 and $200 \mathrm{~nm}$, endocytosis is their main internalization pathway. Endocytosis is an active energy-consuming process that ensures entry of substances into the cells. ${ }^{[182]}$ Although 
numerous studies found out a direct correlation of the increased endocytosis of NPs with the decreasing of their size, ${ }^{[183-185]}$ few studies were focused on the size effect on NEs endocytosis. Besides, most of these studies are difficult to compare because different materials were used. Based on previous reports on NEs, it is clear that their internalization is a complex function of different factors, including size, nature of emulsifiers, carrier oil and interfacial characteristics (surface charges). ${ }^{[186-188]}$ Focusing on size effects, Fan et al. studied the cellular uptake of NEs with three droplet diameters $(170,265$ and $556 \mathrm{~nm}) .{ }^{[189]}$ The uptake efficiency in this study was analyzed based on the fluorescence intensity of loaded dye inside the cells, revealing strong dependence on the nano-droplet size. It allowed the authors to conclude that NEs of smaller size could enter into cells by endocytosis faster than larger NEs. Zheng et al., ${ }^{[185]}$ also showed an increased uptake for lower droplet size of NEs loaded with 5-demethyltangeretin (5DT) (Figure 7). In this study, authors measured the internalization of 5DT into a suspension of HCT116 cells from different media: bulk water, medium chain triglyceride (MCT) and NEs with different sizes (67 nm, $125 \mathrm{~nm}$ and 203 $\mathrm{nm}$ ), after incubation for $0.25,1$, and $4 \mathrm{~h}$. HPLC determination after 5DT extraction from cells suggested that 5DT internalization was highest when delivered in $67 \mathrm{~nm}$ NEs. Taking this condition as a reference $(100 \%)$, the internalization rate for other conditions was $92.9 \%$, $30.6 \%, 5.9 \%$ and $13.1 \%$ in that of $125 \mathrm{~nm}$ NEs, $203 \mathrm{~nm}$ NEs, bulk water and MCT, respectively. The low internalization of 5DT when delivered from bulk water and MCT suggested minimal interference of direct leakage from NEs to cells, highlighting the role of endocytosis of NEs containing 5DT. The effect of nano-droplet size on the internalization was confirmed by fluorescence microscopy of cells internalized with Nile Red-loaded NEs (Figure 7B). In the study by Yi et al., authors found out that smaller droplet size of lipidbased emulsions improved efficiency of a cargo (drug) delivery, defined by the amount of loaded drug recovered in the aqueous phase after ultracentrifugation. ${ }^{[190]}$ Two possible mechanisms have been proposed to explain the effect of size. ${ }^{[191,192]}$ The first one is sizedependent contact curvature: smaller particles have more adhesive contacts with the cellular membrane and less energy is required for deforming membrane around droplets for the formation of the endosome. ${ }^{[192,193]}$ Second one is size-dependent droplet deformability, where smaller droplets exhibit more defined spherical shape with lower deformability and thus can easier enter the cells. ${ }^{[191,192]}$
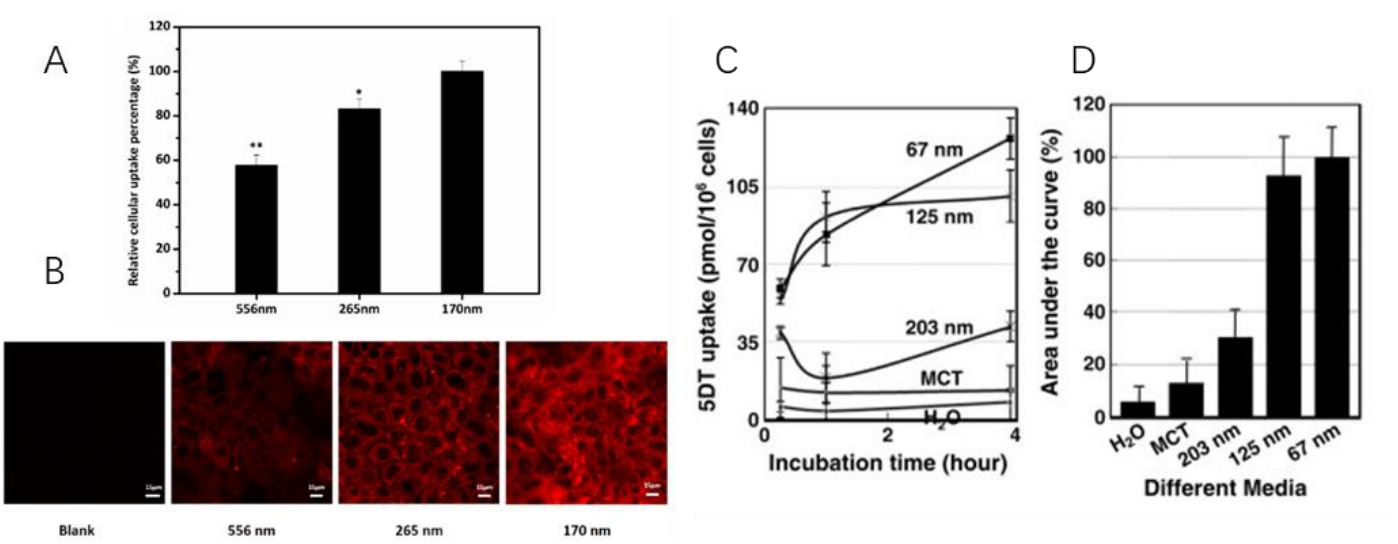

Figure 7. The effects of three different droplet sizes on cellular uptake of lipid-based emulsions (A) Relative uptake of emulsions of different diameters by Caco-2 cells. The 170 NEs were used as control (100\% uptake). Other lipid emulsion sizes were compared to the control using ANOVA (*p $<0.05$, ** $\mathrm{p}<0.01$ ). (B) CLSM of 
Caco-2 cells grown on glass coverslips after exposure to three different droplet size lipid-based emulsions containing Nile red for $4 \mathrm{~h}$. Scale bar indicates $15 \mu \mathrm{m}$. Influence of emulsion delivery system type on the uptake of 5DT detected by HPLC. (C) Intracellular levels of 5DT at different times after incubation with different emulsions. (D) Area under the curve (AUC) of intracellular levels of 5DT after treatment with different emulsions. The data were expressed as mean \pm standard with three independent replicates. Reproduced with permission. ${ }^{[185,189]}$ Copyright 2017 Nanomaterials and 2014 Food Research International.

However, one should also take into account that size of NEs can also influence their stability, especially dye leakage. Indeed, larger NEs with less surface-volume ratio can be more stable in the holding of dyes with lower problem of leakage. Our previous studies showed that the dyes can be released before NEs enter the cells. ${ }^{[42]}$ Indeed, when NEs loaded with Nile Red were incubated with cells, already after 15 min a significant emission was observed inside the cells (Figure 8A,B). By contrast, incubation with NR688-loaded NEs showed no detectable fluorescence signal even after $2 \mathrm{~h}$ of incubation at $\mathrm{rt}$ (Figure 8A,C). These results suggest that NR668 NEs do not enter the cells in these conditions, whereas Nile Red undergoes fast leakage out of the NEs followed by intracellular accumulation. These data, in line with FRET and FCS results for Nile Red (see above), can be explained by insufficient lipophilicity of Nile Red, so that its affinity to the oil core of NEs is not strong enough to be efficiently protected in biological environment. Therefore, great attention should be paid in cellular studies using Nile Red or other medium-lipophilic dyes as the loading cargo for NEs.

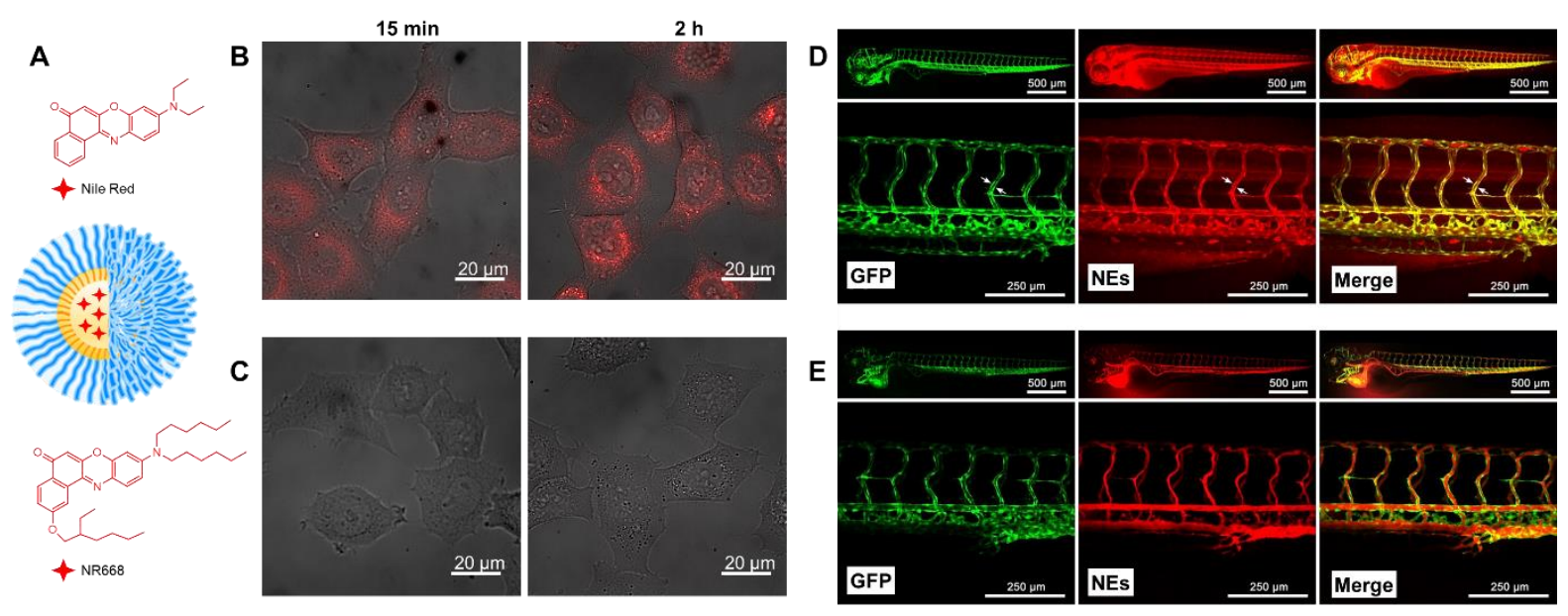

Figure 8. Imaging cells incubated with NEs loaded with Nile Red and its lipophilic derivative NR668. (A) Presentation of NEs loaded with Nile Red or NR668. (B,C) Combined fluorescence and transmission images of HeLa cells incubated with nano-droplets containing 0.1 wt.\% of Nile Red (B) or 1 wt.\% of NR668 (C) for different times: 15 min and 2 h. (D,E) Zebrafish microangiography using NEs containing 0.1 wt.\% of Nile Red (D) or 1 wt.\% of NR668 (E) with the Tg(fli1:eGFP)y1 line. 3 days post fertilization, the living zebrafish embryos were injected $2.3 \mathrm{~nL}$ of nano-emulsions diluted twice in HEPES buffer $(\mathrm{pH} 7.4)$ and imaged with a confocal microscope. Images present the global view (upper panels) and a zoom in of the trunk vasculature (lower panels). The images in green present endothelial cells expressing eGFP, while in red - fluorescence of Nile Red and NR668, 30 min after the injection of the nano-emulsion. The arrows show the endothelial cells. The merged image shows the localization of Nile Red in the endothelium and the global diffuse labeling of the entire embryo, while the merged image shows no colocalization of NR688 with the endothelial cells after 2 hours. Reproduced with permission. ${ }^{[42]}$ Copyright 2012 Biomaterials. 
The absence of signal from NR668-loaded NEs with cells suggested remarkably weak interactions with the cell surface. On the one hand, it can be explained by high deformability of these liquid-core NEs, in line with other studied mentioned above. ${ }^{[191,192]}$ On the other hand, their stealth properties probably stems from their PEGylated shell formed by the non-ionic surfactant, which is known to inhibit non-specific interactions with the cell surface for different NPs. ${ }^{[194-196]}$ To modulate NEs surface chemistry, negatively charged carboxylates were inserted to NEs interface using amphiphilic polymer PMAO. ${ }^{[176]}$ The obtained NEs containing $1 \%$ of PMAO and loaded with NR668 were able to enter the cells in 2 hours, which was not the case of the parent NEs without polymer. Thus, negatively charged carboxylates on the NE surface favor interactions of NEs with cells and their internalization. ${ }^{[176]}$ However, further work is needed on surface chemistry of NEs, in order to control better their interactions with cells.

\subsubsection{Tracking nano-droplets inside cells}

Single particle tracking in cells is a great way to gain insight into the dynamics of intracellular trafficking and other macromolecular behavior. ${ }^{[197]}$ It is also important for understanding how nano-objects interact with the intracellular environment. The most important aspect of single-particle tracking in cells is usually high signal to noise ratio and tracking time. The first method to improve this is usually by using two-photon excitation (TPE) imaging, since cellular contents are generally transparent under infrared excitation wavelength. ${ }^{[198-200]}$ However, it is a scanning technique, which is slower than wide-field single-photon excitation, which can easily provide video rate imaging of single molecules and NPs. ${ }^{[63,112,201]}$ The key approach to improve signal to noise ratio is to use NPs of highest possible brightness. Up to now, most of particle tracking experiments in cells have been done using quantum dots, because they are 10-100 times brighter than single organic dyes and feature high photostability, which allows robust continuous recording of single-particle traces. ${ }^{[202-204]}$ Nowadays, organic NPs started gaining popularity because of improved quantum yield, brightness and controlled small size, which enables them to be tracked with high precision and for longer periods. ${ }^{[205-207]}$

Recently, a novel dioxaborine dye named DBS- $\mathrm{C}_{8}$ was developed and encapsulated into NEs with vitamin E acetate as liquid core (around $40 \mathrm{~nm}$ ) at high concentration of 37 $\mathrm{mM}$ in oil without obvious aggregation caused quenching issue and a high quantum yield of 0.44. ${ }^{[63]}$ Moreover, in comparison to NR668, it showed significantly improved photostability and single-particle brightness. These improved optical properties of fluorescent NEs enabled their single-particle tracking as well as determination of their trajectory and velocity in the cytoplasm (Figure 9). Remarkably, NEs bearing negative surface change due to presence of PMAO polymer at the interface significantly decreased the intracellular mobility of NEs, probably due to increased non-specific interactions with the intracellular components (Figure 9). This successful example shows a strong potential of dye-loaded NEs as a tool for particle tracking analysis and the importance of dye design for achieving desired optical properties. 


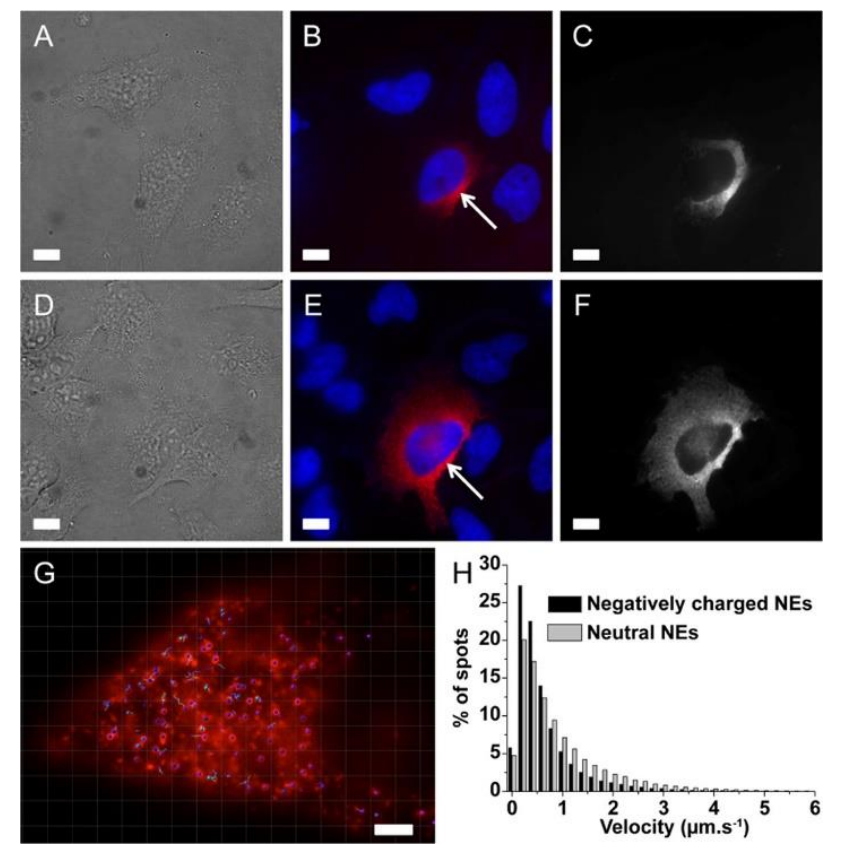

Figure 9. (A) Microinjection of DBS-C 8 -loaded NEs $(41 \mathrm{~nm})$ in HeLa cells and their individual tracking. Images of HeLa cells microinjected with negatively charged NEs (A-C) and neutral NEs (D-F). (A, D) Transmission light microscopy 5 min after injection. (B, E) Epifluorescence imaging 15 s post-injection; the NEs are in red and the nuclei are in blue (Hoechst $5 \mu \mathrm{g} \mathrm{mL}^{-1}$ ); white arrows indicate the injection point. (C, F) NEs' maximum intensity projection of a $30 \mathrm{~s}$ time laps ( $50 \mathrm{~ms}$ acquisition time, a total of 600 images), $5 \mathrm{~min}$ post-injection. Scale bar is $10 \mu \mathrm{m}$. (G) Example of a HeLa cell microinjected with neutral NEs where single NEs have been spotted (blue spots) and their trajectory tracked using Imaris 9.2.1 (Bitplane Inc) software, scale bar is $3 \mu \mathrm{m}$. (H) Histogram of NEs' velocity in the cytosol obtained by individual NEs' tracking from movies right after injection (30 s, 50 ms acquisition time). Reproduced with permission. ${ }^{[63]}$ Copyright 2019 ACS Appl. Mater. Interfaces.

\subsubsection{Cytotoxicity}

To understand the cytotoxicity of dye-loaded NEs, it is crucial to investigate the cytotoxicity of NEs itself. Among the two components, oil and surfactant, the latter can be a source of toxicity, because surfactants are known to provide multiple toxicity effects on cells and they are the part of NEs directly in contact with cells. ${ }^{[08]}$ In this case, the earlier studies on drug-loaded NEs shed light on the cytotoxicity of NEs without dyes. Yoon et al. focused on the effect of surfactant of NEs cytotoxicity in different cell lines (NIH3T3, H9C2, HepG2, hCPC, and hEPC). NEs with average diameter of $200 \mathrm{~nm}$ were formulated for delivery of curcumin, which is a natural compound with multiple therapeutic activities, including anti-cancer and anti-inflammatory. Authors prepared two different types of NEs with different ratio of synthetic surfactant Tween 80 and of natural emulsifier (lecithin). Without drug, both types of NEs did not show any detectable cytotoxicity, which can be related to low concentrations $(2.5 \mathrm{mg} / \mathrm{mL}$ of oil) of NEs were tested. Moreover, formulations of curcumin with NEs significantly reduced its cytotoxicity in cells, probably because NEs decreased access of the drug to cells. ${ }^{[209]}$ Vater et al. compared the cytotoxicity of lecithinbased NEs with NEs based on conventional surfactants on human skin cells (primary human keratinocytens as well as fibroblasts). In addition, they evaluated the effect of the nanodroplet size in the range from $124 \mathrm{~nm}$ to $245 \mathrm{~nm}$. Authors made the NEs concentration 
extremely high $(75 \mathrm{mg} / \mathrm{mL}$ of oil) in order to have a more obvious effect by NEs themselves in the cell medium. MTT assay for primary human keratinocytes cells showed that NEs based on anionic and non-ionic surfactants (SDS, SLES, APG and Tween 80) showed strong cytotoxicity (nearly $0 \%$ viability) after $24 \mathrm{~h}$ incubation. In sharp contrast, all the NEs samples prepared by using lecithin-based surfactant showed high cell viability ranging from 34 to $50 \%$ depending on the formulation. This result clearly shows that synthetic surfactants whether ionic or not have higher cytotoxicity compared to natural lipids. However, the effect depended strongly on the cell line, as the difference of cytotoxicity for fibroblast cells was much less pronounced, all the formulations led to a mean cell viability of $82.43 \%$ $( \pm 11.83 \%)$, indicating that any comparison of cytotoxicity of different formulations should be done for a given cell line. Overall, these results suggested that replacing synthetic surfactants with natural surfactant lecithin is beneficial for reducing the cytotoxicity of NEs used at high concentration. However, at lower concentrations $(5 \mathrm{mg} / \mathrm{mL}$ of surfactant) NEs did not show any clear cytotoxic independently of the nature of their surfactant. ${ }^{[209,210]}$ Our previous data showed that in case of NEs formulated with GRAS components (labrafac and Kolliphor $^{\circledR}$ ELP), the cytotoxicity is observed only at relatively high concentrations (7.5 $\mathrm{mg} / \mathrm{mL}$ surfactant and $7 \mathrm{mg} / \mathrm{mL}$ oil).

Dye loading inside NEs usually does not contribute to increase the cytotoxicity of NEs, because its concentration in the oil rarely exceeds $1 \mathrm{wt} \%$. In our previous studies, even heavy loading ( $8 \mathrm{wt} \%$ ) with cyanine dye and its bulky counterion (DiI-TPB) did not significantly affect the cytotoxicity of NEs. Remarkably, cytotoxicity of dye-loaded NEs remained very similar for most of NEs formulations, except the highest concentration (21 $\mu \mathrm{M}$ DiI-TPB dye concentration, $15 \mathrm{mg} / \mathrm{mL}$ oil). ${ }^{[112]}$ However, the latter dye concentration was >20-larger than that normally used in imaging experiments, indicating that these dyeloaded NEs can be safely used for bioimaging at the cellular level.

Nevertheless, when doing cell imaging experiments with dye-loaded NEs, attention should be paid to the phototoxicity of dyes. Cells observed under fluorescence microscopy may be subject to photo-damage due to the production of light-induced reactive oxygen species (ROS), especially when fluorophores are present. ${ }^{[211]}$ Fluorophore in excited state can react with oxygen to generate singlet oxygen that can disrupt the redox homeostasis of the cells and result in physiological damage of the cells, thus decreasing the quality of cell imaging results. Even without added fluorophores, ultraviolet light is harmful for mammalian cells. High excitation power used in new imaging techniques could be also problematic. Indeed, cellular imaging with maximal signal/noise ratio and resolution usually needs high excitation light irradiance. ${ }^{[212]}$ Moreover, it is commonly accepted that shorter wavelength of light is more toxic for cell samples. The problem of phototoxicity is generally avoided by applying less irradiance or shorter illumination period. Normally, before the imaging experiment, one should determine the illumination irradiance that generate phototoxic effect, and work well below this irradiance threshold to prevent potential phototoxic effects. ${ }^{[213]}$ An alternative option is to encapsulate dyes absorbing in the red to near-infrared optical window, where the photo-damage is generally lower. ${ }^{[214,215]}$ However, one should take into account that current optical microscopes are optimal in the visible 
spectral range, so that optical imaging with NIR dyes requires higher excitation power. The optimal spectral window for microscopy is between green $(500 \mathrm{~nm})$ to red $(650 \mathrm{~nm})$.

Overall, photo-toxicity effects of NEs loaded with fluorescent have not been sufficiently investigated. Although cytotoxic effects of free drug and drug loaded NEs were often compared, no comparative study has been conducted about the photo-toxicity effect of highly lipophilic dye to cells in its free form and NEs encapsulated form. This would be an interesting aspect to investigate in the future, whether NEs can provide a photo-protective effect on the encapsulating dyes. On the other hand, photo-toxicity of NEs loaded with photosensitizing dyes, specially designed for singlet oxygen generation have been studied for photodynamic therapy (see chapter 4).

\subsection{In vivo applications}

NEs are particularly attractive for in vivo imaging and delivery applications in comparison to small organic dyes (cargos) because of their prolonged circulation periods, as well as the ability to encapsulate different cargos, both contrast agents and drug molecules. ${ }^{[19]}$ The fate of NEs in vivo, their bio-distribution and their disintegration process have been intensively studied in the last decade.

\subsubsection{Dye-loaded NEs in Zebrafish embryo}

The zebrafish embryo is becoming a powerful vertebrate model for quantitative optical imaging, owing to its high transparency in the embryonic and larval stages. ${ }^{[216]}$ Recently, it also appeared as promising in vivo model for studying and treating diseases, especially cancer. ${ }^{[217,218]}$ The application of dye-loaded NEs in zebrafish was initially reported by us using NR668 dye as non-leaking cargo. ${ }^{[2]}$ The NEs were injected into the heart of the embryo followed by live imaging of their bio-distribution. The experiments were done in a zebrafish transgenic line expressing eGFP specially in the endothelial cells for delimiting the vascular network of the embryo. The two-color imaging allowed co-localizing the vascular network in green channel with dye-loaded NEs in Red channel. We found out that NR668 loaded NEs were localized exclusively in the blood circulation system without any signs of dye leakage (Figure 8E). In sharp contrast, Nile Red-loaded NEs showed intense fluorescence in the epithelial cells of vessels and diffused fluorescence all over the embryo, showing clearly that this dye can rapidly leak out of NEs and distribute all over the embryo (Figure 8D). This study on one hand, validated NEs as promising tool for imaging blood circulation of zebrafish. On the other hand, it showed that it is very important to consider the problem of proper choice of dye cargo, in order to avoid in vivo imaging artifacts. Later study found that strong illumination NR668 NEs can trigger release of their content in biological media. This light-controlled cargo release was also tested in zebrafish embryo and it was found that after 5 min illumination through a bandpass filter (450-490 $\mathrm{nm}$ ), fluorescence of NR668 was clearly observed outside of the blood vessels, which was not the case of non-illuminated zebrafish. ${ }^{[142]}$ These experiments, provide a proof-of-concept for the light-controlled release of a dye cargo from NEs in vivo. 
In the further study, we aimed to boost the brightness of nano-droplets in order to track single particles directly in the blood stream of zebrafish. To this end, we loaded 90-nm NEs with DiI with bulky counter-ion TPB (DiI-TPB, Figure 10A) at $8 \mathrm{wt} \%$, which corresponded to $\sim 10,000$ dyes per particle. At normal dilution before injection (1000-fold) these NEs allowed imaging blood circulation of zebrafish without noticeable dye leakage (Figure 10B). At much higher dilution $\left(10^{6}\right.$-fold), these ultra-bright NEs enabled first single-particle tracking in live zebrafish embryo. (Figures 10C,D). ${ }^{[12]}$ The tracking analysis showed strong variation in the single particle velocity produced by a heartbeat of the embryo, which matched well with the velocity of blood cells (Figures 10E-G). Single-particle tracking in vivo opens new horizons in bioimaging and nanomedicine, because it enables monitoring directly individual nanocarrier, and thus understand better bottlenecks in the field of drug delivery. ${ }^{[219]}$

A

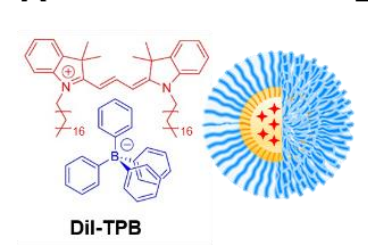

C Tracking

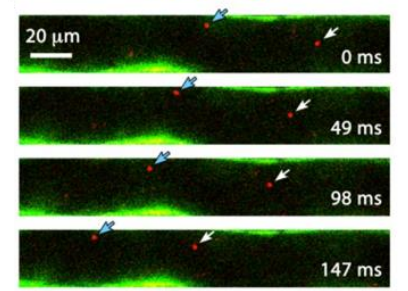

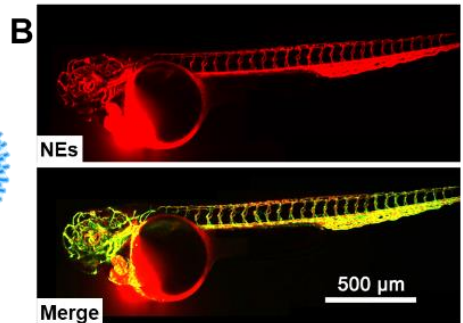

D Line analysis

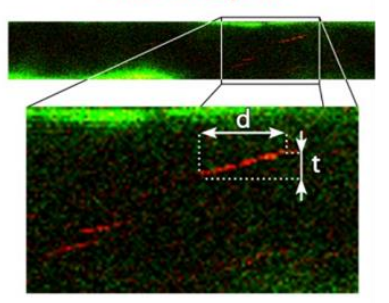

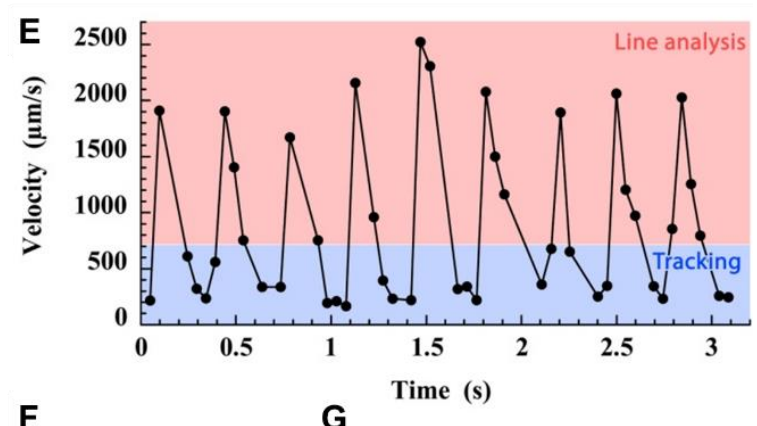

G

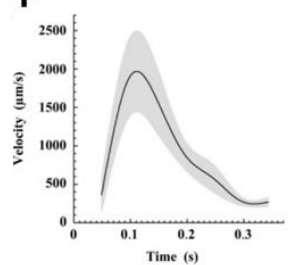

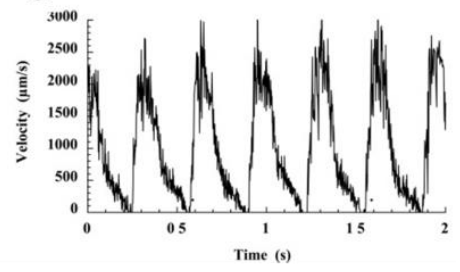

Figure 10. Zebrafish microangiography and single-particle tracking using 90-nm nano-droplets containing 8 wt.\% of DiI-TPB injected in $T g(f l i l: e G F P)^{y l}$ embryos 3 days post fertilization. (A) Scheme of NEs loaded with DiI-TPB. (B) Images present the global view of zebrafish in the DiI-TPB NEs channel (top), 30 min after injection, and merged image with GFP channel for endothelial cells expressing eGFP (bottom). (C-G) Singleparticle tracking of DiI-TPB NEs in zebrafish vessels. (C) During the diastole single particles were followed in consecutive frames. (D) During the systole, the velocity was calculated from the shape of the line, which is a result of the movement of the particles during the line scanning. (E) Reconstruction of the blood flow profile. The velocities during the diastole (lower part $<500 \mu \mathrm{m} / \mathrm{s}$ ) were determined by manual tracking (C) while the peak velocities $(>500 \mu \mathrm{m} / \mathrm{s})$ were deduced from the line profiles (D), where $\mathrm{d}$ and $\mathrm{t}$ define the particle displacement and the required time, respectively. (F) Average velocity profile of 10 pulses shown in panel E. (G) Example of velocity profile of the blood flow in the dorsal aorta determined by tracking the blood cells. Adapted with permission. ${ }^{[12]}$ Copyright 2014 Biomaterials.

\subsubsection{Dye-loaded NEs used for mice imaging}

Imaging of NEs in mice is very important, because mice is a key model animal used in cancer research and therapy. ${ }^{[220]}$ However, unlike zebrafish embryo, imaging of mice is strongly altered by auto-fluorescence and light-scattering of tissue and blood. ${ }^{[221,222]}$ These interferences can be decreased by using dyes operating in the NIR region (> $700 \mathrm{~nm})$, where those signals from tissue are minimal. ${ }^{[214,223,224]}$ The key question related to in vivo 
applications of NEs is whether they are able to keep their cargo after injection up to reaching target (e.g. tumor). Indeed, the nanomedicine has been established mainly on solid organic and inorganic NPs, ${ }^{[225,226]}$ while NEs, having liquid core, has not been considered as a carrier with sufficient stability. Since more deformable particles tend to be less prone to cellular uptake, ${ }^{[191,192]}$ NEs with a liquid core can present minimal non-specific internalization into cells (see above) and potentially longer blood circulation time. On the other hand, liquid core NEs raises questions about stability of NEs in vivo. As mentioned above, the key tool to understand integrity of a NEs and the leakage of cargo is FRET between two encapsulated dyes. This approach has already been used to study integrity of different NPs in vivo. ${ }^{\text {[227-229] }}$ For instance, Hammond et al. reported a micelle polymer chemically modified with two azide-containing near-infrared (NIR) cyanine dyes named Cy 5.5 and Cy 7 by click chemistry. ${ }^{[229]}$ The integrity of this polymer particle was monitored by the FRET efficiency of these two dyes to study the in vivo fate of this polymer nanoparticle after injection in the blood circulation system of mice.

One of the first attempts to generate FRET NEs for in vivo imaging was done by Texier et al., where red DiD was combined in a FRET acceptor NIR dye DiR. ${ }^{[165]}$ These NEs enabled monitoring biodistribution of NEs in vivo, showing their remarkable stealth character. However, as the FRET signal was relatively weak, it was difficult to quantify the integrity of NEs in vivo. Later on, we designed FRET NEs by loading donor (Cy5.5LP) and acceptor (Cy7.5LP), both operating in the NIR region (Figure 11A, B). Efficient FRET was achieved by improving dye loading to $1 \mathrm{wt} . \%$ with help of bulky hydrophobic counterion TPB (Figure 11C). Once injected at the tail of mice, donor and acceptor emission was detected by the whole animal imaging setup by exciting the donor dye (Figure 11D). ${ }^{[113]}$ At the initial injection time, the donor intensity was much lower than the acceptor channel all over the healthy mice. Over time, donor channel intensity increased, while the acceptor intensity dropped, reflecting the loss of FRET over time (the FRET ratio decreased over time with pseudo-color switching from red to cyan), reflecting disintegration of NEs (Figure $11 E$ ). Using calibration solutions of NEs under the same imaging setup up it was possible to establish a calibration of the FRET ratio and thus directly quantify the integrity of NEs in mice (Figure 11D). Remarkably, emission from tail vein revealed that the integrity of these FRET NEs in the blood circulation of healthy mice was preserved at $93 \%$ at $6 \mathrm{~h}$ of postadministration (Figure 11G). On other hand, integrity of NEs dropped faster in liver, i.e. to $66 \%$ after $6 \mathrm{~h}$ (with half-life of $8.2 \mathrm{~h}$ ). Experiments in tumor bearing mice showed that these NEs accumulated efficiently in tumors (Figure 11F) being nearly intact (77\% integrity at 2 h) and then decreased their integrity to $40 \%$ at $6 \mathrm{~h}$ (with half-life of $4.4 \mathrm{~h}$ ). This study proposed a robust FRET-based methodology to evaluate quantitatively the nanocarrier integrity in small animals. The results also showed that NEs are remarkably stable, remaining nearly intact in the blood circulation and reaching the target tumor in nearly intact form. The latter validate them as prospective nanocarriers for drug delivery applications. 

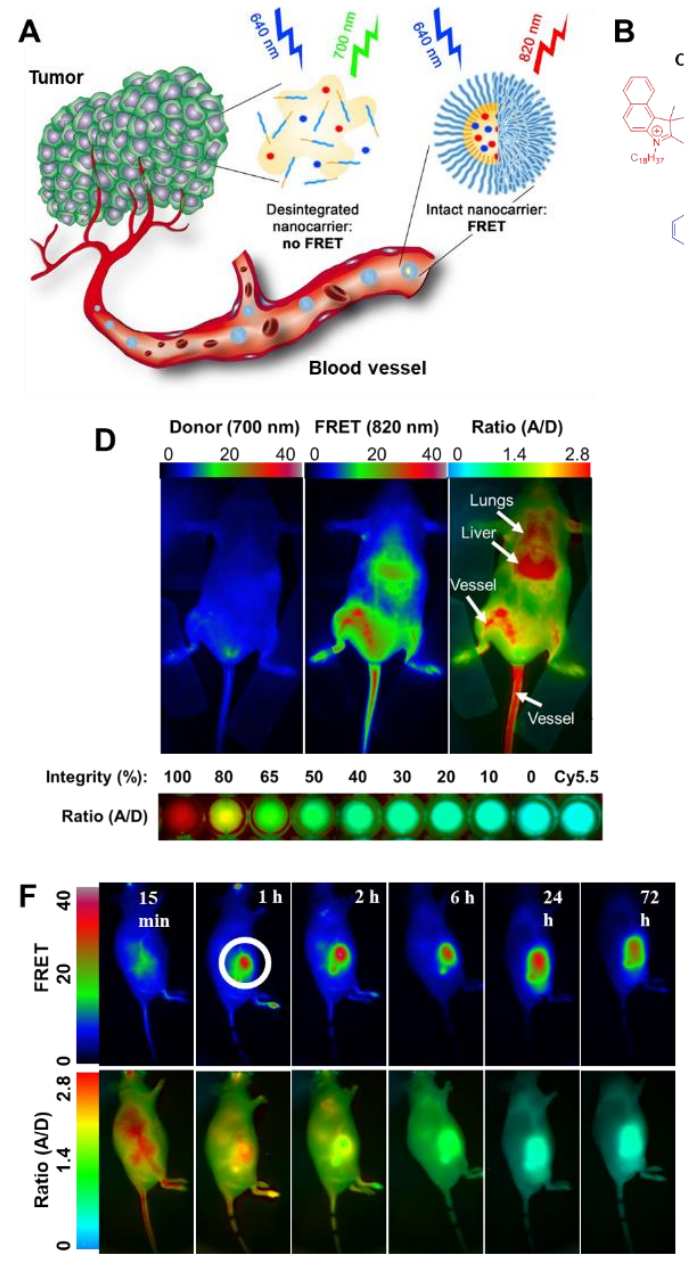

B
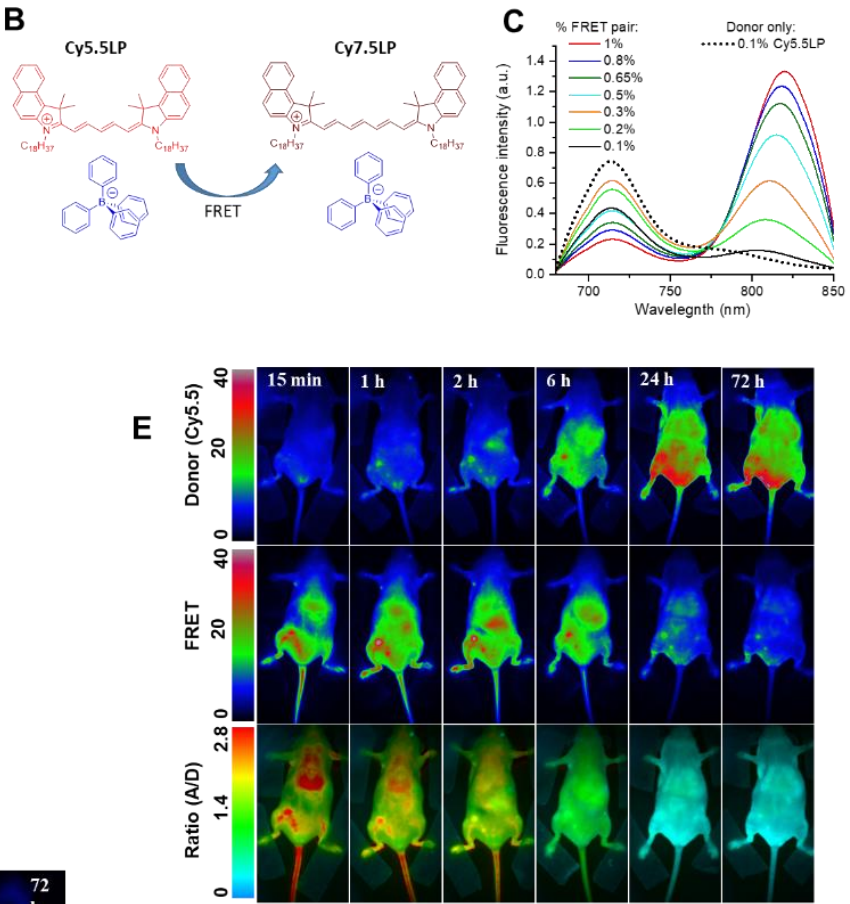

G

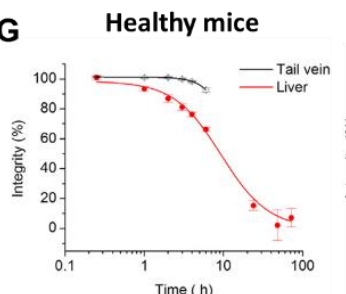

Tumor-bearing mice

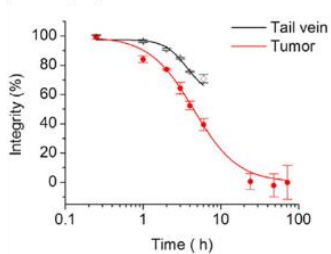

Figure 11. Concept of FRET NEs that can report on their integrity by change in their emission color (A) and the NIR dyes with their hydrophobic counterion (Cy5.5LP and Cy7.5LP) used for encapsulation (B). (C) Fluorescence spectra of dye-loaded NEs as a function of weight $\%$ of FRET pair (corresponds to \% of each dye). (D) FRET imaging of healthy nude mice $15 \mathrm{~min}$ after injection of NIR-FRET NEs (1\% of Cy5.5LP and Cy7.5LP each). Low panel (D) shows calibration of the imaging setup, where the ratio (A/D) image corresponds to level of integrity of NEs. (E) FRET imaging of healthy nude mice at different times after injection with NIR-FRET NEs and control NEs containing only Cy5.5LP dye (1\%). Upper panels present intensity images of the Cy5.5LP channel $(700 \mathrm{~nm})$, middle panels present images of Cy7.5LP channel (820 $\mathrm{nm}$ ), while the lower panels present ratiometric images (acceptor/donor). The excitation wavelength was 630 nm. (F) FRET imaging of tumor-bearing nude mice at different times after injected with NIR-FRET NEs. (G) Analysis of NEs integrity in different regions of healthy and tumor-bearing mice as a function of postadministration time. Reproduced with permission. ${ }^{[113]}$ Copyright 2016 J. Control. Release.

In addition to systemic administration, fluorescence imaging can help to understand the fate of lipid nanocarriers after oral gavage administration, which is used in the oral drug delivery. In this case, highly hydrophobic dyes, which undergo quenching in water through ACQ was used for encapsulation in solid lipid nanoparticles (SLNs). (Figure 12A). ${ }^{[230]}$ This quenching phenomenon was exploited to extract information about the integrity of SLNs in vivo. This was achieved by comparing fluorescence intensity of quenchable BODIPY cargo with non-quenchable dye DIR (Figure 12). The quenchable probe showed significant time dependent loss of fluorescence in the intestinal tract related to SLN digestion (disintegration), whereas DIR loaded SLNs did not show significant intensity change. This in vivo imaging suggested predominant disintegration of SLNs within $2 \mathrm{~h}$ and complete 
digestion within $4 \mathrm{~h}$, which correlated well with the in vitro lipolysis data. The described methodology of water-quenching fluorescence dyes can be used as probes to monitor the in vivo fate of lipid-based nanocarriers after oral administration. So far, in vivo imaging of NEs after oral delivery in mice has not been realized to date. But these experiments would be of particular interest because both mode ${ }^{[166,231]}$ and animal studies ${ }^{[232]}$ show the great potential of NEs as drug delivery agent through the intestinal route.
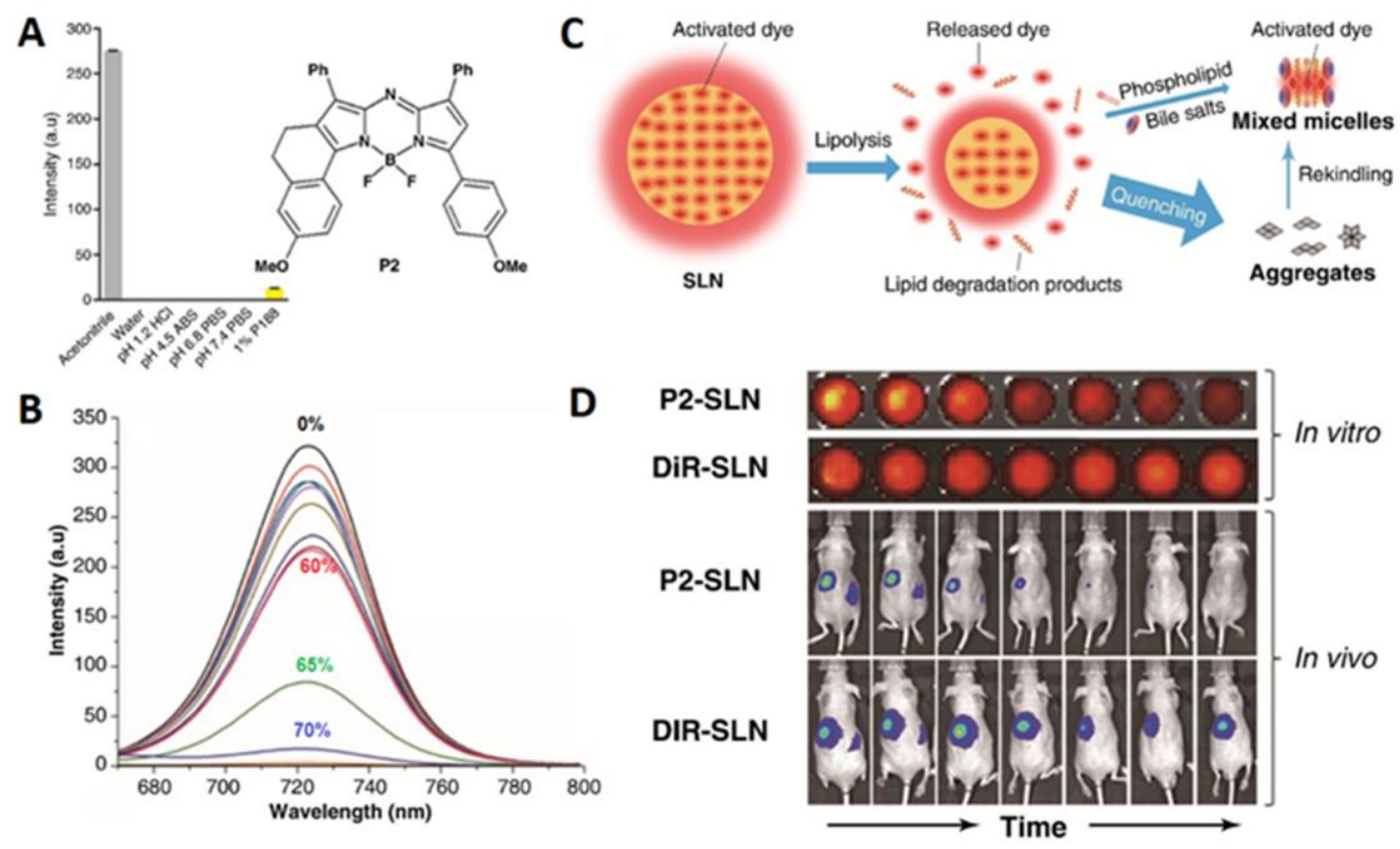

Figure 12. Schematic representation of the rationale of exploring the in vivo fate of lipid-based nanoparticles using environment-responsive aggregation-caused quenching (ACQ) probes. (A) The water sensitivity and chemical structure of a model ACQ dye P2; (B) Fluorescent emission spectra in response to the water content (with acetonitrile) in the system; (C) the rationale of exploration of in vivo fate of solid lipid nanoparticles (SLNs); (D) in vitro and in vivo imaging of P2-labeled SLNs in comparison with a conventional dye DiR. Reproduced with permission. ${ }^{[230]}$ Copyright 2015 Nanomed. Nanotechnol.

\subsection{Targeted imaging with dye-loaded NEs}

\subsubsection{Passive tumor targeting}

It is established that the abnormal angiogenesis of tumor tissues produces porous blood vessels and permeable holes that could facilitate the internalization of macromolecules and particles. ${ }^{[233]}$ Additionally, the instability of lymphatic vessels in tumor areas might enhance the retention time of drugs, macromolecules and particles since their clearance rate decreases, so that they are more likely to be retained in the tumor tissue. ${ }^{[234,235]}$ This phenomenon is called the enhanced permeability and retention (EPR) effect.

Based on this effect, dye-loaded NEs with size between 20-100 nm, being large enough to avoid fast renal clearance, but small enough to pass the blood vessels, are an attractive agent for the monitoring of the EPR directed accumulation of NEs (drug-loaded or not) in tumors. For instance, Radicchi et al., observed the developing stages of NEs 
accumulation in tumor areas based on a DiR loaded NEs. Authors observed some increase fluorescence intensity of DiR of dye-loaded in NEs in comparing to DiR administrated alone, indicating that NEs favors accumulation of dye cargo in tumor areas. This effect became successively more obvious in 4 weeks during the experiment. ${ }^{[236]}$ Jacquart et al. reported a 50-nm NEs loaded with lipophilic NIR dye, derivative of IR780. Owing to their sufficiently long circulation time on the time scale of hours, these NEs enabled passive targeting and imaging of human prostate cancer cells implanted in mice. ${ }^{[16]}$ It is also reported by Juliette et al. that a lipophilic dye-loaded NEs with diameter of $55 \mathrm{~nm}$ exhibited the preference to accumulate in tumors. ${ }^{[237]}$ However, in this study, authors also observed fluorescence from some organs (especially liver) involved in steroid hormone synthesis and storage. Authors attributed the reason to the fact that NEs itself being lipid based nanoparticle has a higher affinity for areas rich in the steroid hormones. Since lipoproteinreceptor is overexpressed in cancer cells of hormone-dependent tumor areas, it could be used as another feature for passive targeting of dye-loaded NEs (Figure 13). Our studies with FRET NEs loaded with NIR dyes Cy5.5LP and Cy7.5LP also showed an EPR effect, with strong accumulation in tumor already after $1 \mathrm{~h}$ post-administration in tumor bearing mice (Figure 11F). Remarkably, imaging of dissected organs revealed that fluorescence signal from tumor was the largest followed by liver and then lungs, while practically no signal was observed in other organs. ${ }^{[113]}$
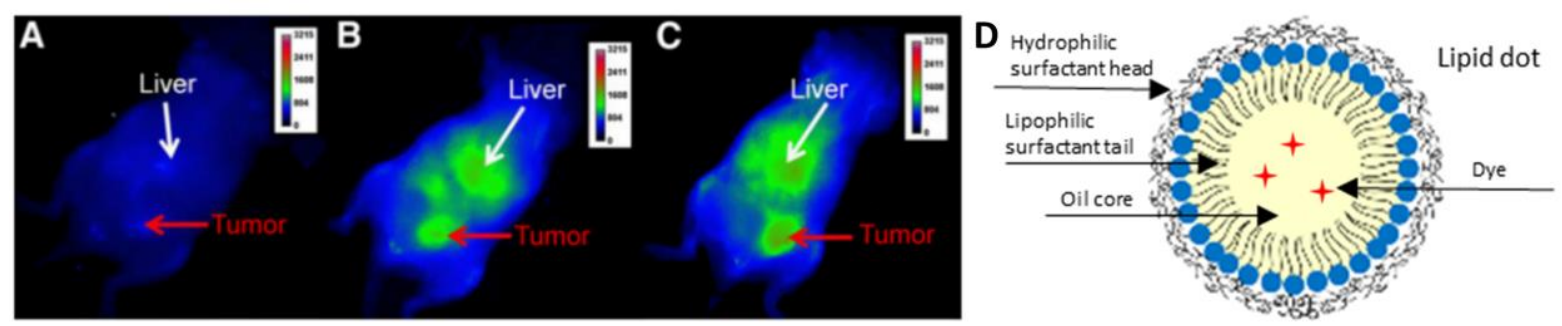

Figure 13. Uptake of DiD-loaded lipidots in PyMT mammary cancer cells. Fluorescence images were obtained before injection (A), $5 \mathrm{~h}$ after injection (B), and $24 \mathrm{~h}$ after injection (C). (D) Schematic presentation of lipid dot is shown. Reproduced with permission. ${ }^{[237]}$ Copyright 2013 J. Nucl. Med.

\subsubsection{Active targeted imaging of tumors}

For targeted imaging, it is necessary to conjugate a nanoparticle with targeting ligands, which include small molecules (e.g. folate, RGD, etc) and biomolecules (antibodies or aptamers). However, as the chemical functionalization of NEs is rather challenging because of liquid nature of these particles, the examples of NEs bearing targeting ligands are still limited. In one approach, bovine serum albumin (BSA) conjugated with folic acid was used to formulate NEs by high pressure homogenization. ${ }^{[238]}$ Owing to hydrophobic and hydrophilic domains, BSA served as a surfactant located at NEs' interface exposing the ligand. Fluorescence labelling of NEs was also provided though BSA using fluorescein derivative (FITC). Folate-tagged protein NEs were shown to selectively internalize into folate receptor-positive cells.

The second approach, which is the most commonly used for NEs, is functionalization of PEGylated lipids or surfactants. In a previous study, DSPE-POE5000-maleimide was used 
to formulate NEs, ${ }^{[172]}$ followed by grafting by maleimide-thiol coupling of the targeting cyclic RGD-based ligand (cRGD-SH) as well as control ligands (cRAD-SH, OH-SH) (Figure 14A). In order to track these NEs, they were loaded with DiD in the oil phase. Authors found significant fluorescence intensity difference between targeting group (LNPcRGD) with non-targeting groups (LNP-cRAD and LNP-OH) from fluorescence cell imaging results and flow cytometry analysis (Figure $14 \mathrm{~B}-\mathrm{C}$ ). Later on, in another research, the capacity of these RGD-functionalized NEs to target cancer cells HEK293( $(\beta 3)$ overexpressing target receptor was evaluated in vivo on mice. It was found that after $24 \mathrm{~h}$ of injection, the fluorescence from ROI areas was much higher in the groups treated with ligand-bearing NEs in comparison to control non-targeted NEs. The results suggested the targeting effect of the ligand that favors accumulation of NEs in cancer cells overexpressing corresponding receptor (Figure $14 \mathrm{D}-\mathrm{F}$ ). ${ }^{[172]}$ These exciting results showed a possibility to enhance the capacity of NEs to accumulate in tumors. However, drug delivery with ligand bearing NEs for anti-cancer therapy remains to be realized. High specificity to the target can be achieved by grafting of antibodies (Abs). Finally, only a rare report shows functionalization of NEs with antibodies, which was also realized using PEGylated lipid with a maleimide function (SPE-PEG3400-Maleimide). The obtained NEs bearing scFv-Fc TEG4-2C antibodies and loaded with iron oxide nanoparticles enabled direct MRI imaging of atherosclerosis in mice models. ${ }^{[239]}$ The development of dye-loaded NEs bearing antibodies for targeted optical imaging constitutes a promising research direction.

One should note that the idea of active tumor targeting by modifying the NEs or NPs surface does not always work as expected. The problem can be associated with the Tumor/Skin (T/S) ratio of specific tumors. ${ }^{[240]}$ In cases when T/S ratio is low, EPR effect could surpass the effect of active targeting in vivo. For instance, Coll and co-workers designed a functional IR780 dye-loaded NEs with surface modified by Arg-Gly-Asp (RGD) peptide, and showed their capacity to target tumor cell overexpressing $\alpha v \beta 3$ integrin. ${ }^{[173]}$ Later on, the authors wanted to verify the generality of this targeting NEs for other tumors. Unfortunately, this effect was not universal: although authors found significant accumulation of this NEs on the membrane of HEK293( $\beta 3$ )-avRFP cells in vitro, a negative result of improving accumulation in DU145 tumor in mice was observed comparing with NEs without this targeting agent. This was attributed to the fact that DU145 expressed insufficient amount of av $\beta 3$ integrin (T/S ratio of 1.5), compared to HEK293(b3)-avRFP cells (T/S ratio of 3.0), ${ }^{[240]}$ so that EPR effect surpassed the specific targeting in the former case. Thus, the targeting effect strongly depends on the cell line, so that the studies on targeted delivery should be systematically tested on multiple cells lines with varied receptor expression.

The ultimate application goal of dye loaded NEs would be the imaging drug-loaded NEs for the treatment of cancer. Systematic efforts should be done in these direction, which could further validate NEs as reliable imaging and drug delivery agents. 


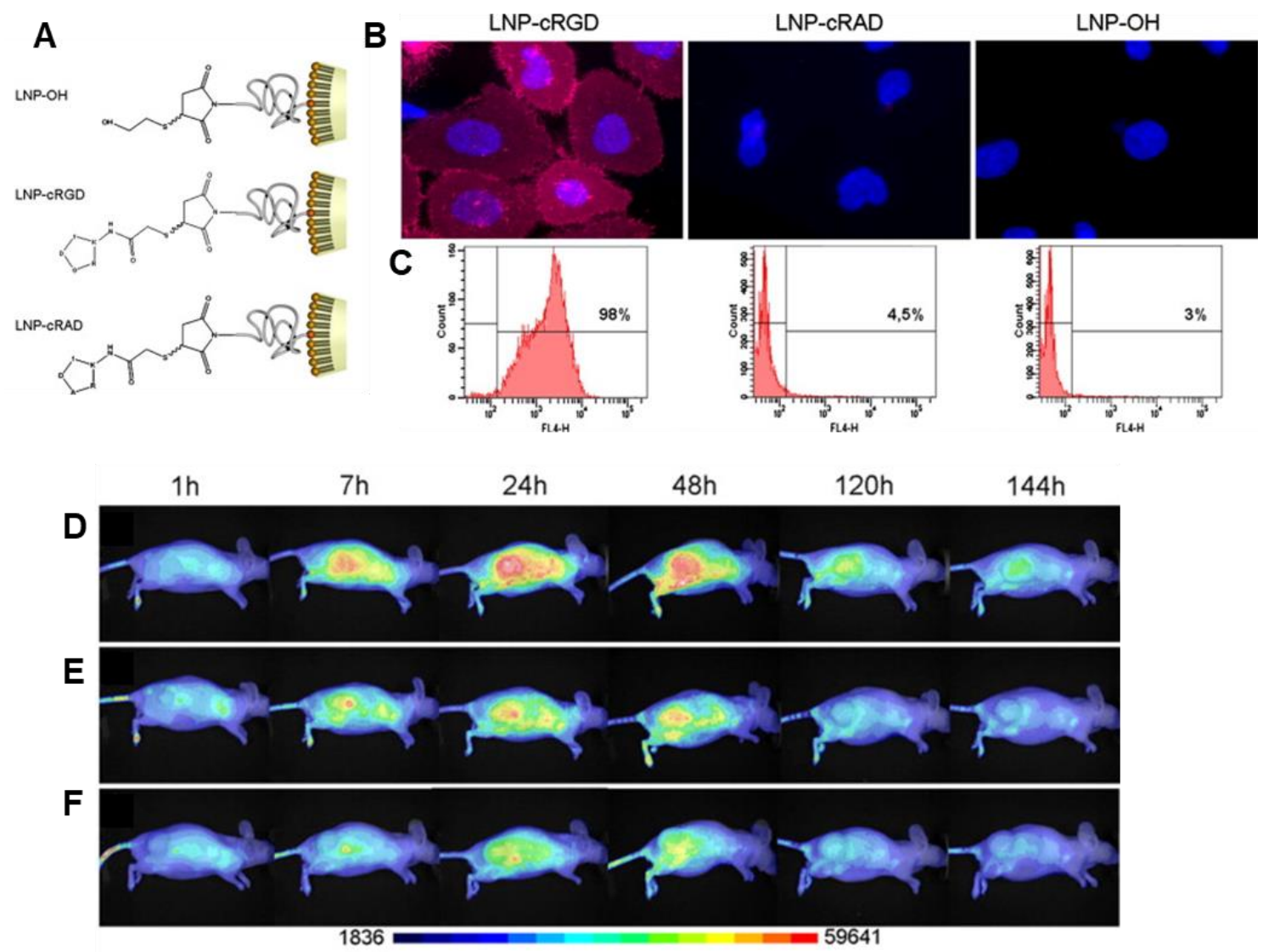

Figure 14. (A) Lipid nano-droplets LNP-OH, LNP-cRGD, or LNP-cRAD prepared by derivatization of maleimide groups at the particle surface. $(\mathrm{A}, \mathrm{B})$ In vitro evaluation of LNP-cRGD, LNP-cRAD, LNP-OH $(0.2$ $\mu \mathrm{M}$ DiD, $141 \mu \mathrm{g} / \mathrm{mL}$ of lipids) in the presence of HEK293( $\beta 3)$ cells after 15 min incubation at $4{ }^{\circ} \mathrm{C}$ in the presence of $10 \%$ fetal bovine serum. Fluorescence microscopy images (A) and flow cytometry analysis (B) evidence the specific LNP-cRGD (red) binding (A and B), in comparison with negative control LNP-cRAD and non-functionalized LNP-OH. The cell nuclei are labeled in blue (Hoechst) on the fluorescence microscopy photographs (B). The figures on the flow cytometry plots $(\mathrm{C})$ represent the percentages of labeled cells. (D-F) In vivo injection of LNP-cRGD (D), LNP-cRAD (E), or LNP-OH (F) (10 nmol DiD, $7.1 \mathrm{mg}$ of lipids dispersed in $200 \mu \mathrm{L}$ ) in HEK293( $\beta 3)$ xenografted Nude mice. Fluorescence images (200 ms integration time, color scale with contrast fixed between 1836 and 59,641) are recorded at different times after injection and superimposed to visible light images (in white and black) (D-F). Reproduced with permission. ${ }^{[172]}$ Copyright 2010 Eur. J. Pharm. Biopharm.

\subsubsection{NEs used as nano-carrier for photodynamic therapy}

Being able to target passively or actively tumor areas grants NEs to be promising nanocarriers for different drugs in order to improve their efficacy. To make the treatment more specific, an optical stimulus could be used for specific drugs which could only be toxic when excited. Photodynamic therapy (PDT) drugs can achieve this purpose. The basic principle of this therapy is the delivery a light sensitive molecule, so-called a photosensitizer (PS), into cells and tissues and further application of the light irradiation. Reactive oxygen species (ROS), generated by PS, produce damage to the targeted diseased cells. ${ }^{[241]}$ PS molecules of the first generation were chemically modified porphyrinoids extracted from blood. Although good PDT effects were observed in these molecules, their application was hindered by the difficulty of their purification as well as their strong retention in tissues, such as skin. One more issue was the short absorption wavelength (around 630 $\mathrm{nm}$ ) characterized by poor light tissue penetration. Higher chemical purity and lower skin retention 
period were achieved for the second generation of PS molecules. ${ }^{[242]}$ Since many years PDT has become a reliable technique for treating certain cancers in clinics, especially those where medical doctors can easily access with an excitation light.

The emerging research about dye-loaded NEs provides a great opportunity for the development of the third generation of PS by encapsulation them inside of NEs core, which are released only after reaching the targeted areas. Moreover, by limiting the light source only to the precise pathology anatomical sites, the damage to other contaminated healthy sites could be avoided. Usually, the applied PS is fluorescent itself, so the location and concentration of this compound could be determined by the fluorescence intensity under suitable excitation wavelength. Meanwhile, the concentration of the generated ROS could be measured by using an appropriate ROS probe. For instance, Park et al. encapsulated a PS named Chlorin e6 (Ce6) in NEs stabilized by mPEG-PCL surfactant and used these NEs for tumor targeting delivery and PDT treatment. Firstly, cellular uptake study was conducted to verify that Chlorin e6-loaded NEs can improve delivery of PS into cells, which can generate more ROS species in comparison to its free PS form. Indeed, after $2 \mathrm{~h}$ incubation NEs formulation showed stronger fluorescence intensity of Chlorin e6 and higher ROS generation monitored by probe 2',7'-dichlorofluorescein diacetate (DCFDA) (Figure 15). The formulation also showed good phototoxicity in vitro on cancer cell lines. Then, in vivo imaging experiments were conducted in mice showing EPR-based accumulation of the NEs in the tumor, in line to capacity of NEs of passive tumor targeting mentioned above. ${ }^{[243]}$ In another study, aluminiumphthalocyanine chloride was used as PS encapsulated inside NEs. ${ }^{[244]}$ Cellular uptake of PS-loaded NEs was confirmed by fluorescence imaging, whereas the changes in the absorbance of the ROS probe (1,3-diphenylisobenzofuran) was used as the indicator for ROS generation ability. It is interesting that at $0.1 \mu \mathrm{M}$ loading, $4.4 \mathrm{~J} / \mathrm{cm}^{2}$ power excitation for 15 min could result in a great decrease of over $80 \%$ cell viability, compared to the cell treated in the dark. In a study by Matos $e t$ al., a well-known PS curcumin was loaded in NEs and efficiently delivered inside cells according to fluorescence microscopy on three different cell lines. The authors showed low toxicity of Curcumin in NEs below the concentration of $20 \mu \mathrm{M}$ (NEs concentration of $8 \mathrm{mg} / \mathrm{mL}$ oil) without radiation (80\% viability) and strong toxicity when excited in a modest excitation power of $80 \mathrm{~J} / \mathrm{cm}^{2}(7 \%$ viability). ${ }^{[25]}$ In this study, NEs also showed protective effect for cells in darkness by comparing the toxicity of curcumin in NEs and its free form. The effect of light-triggered cell death with help of PS-loaded NEs is illustrated by fluorescence imaging in a study of Day et al. ${ }^{[246]}$ The authors used a cell viability label NucGreen that label exclusively dead cells by green fluorescence, and thus differentiate dead from live cells. By applying NEs loaded with fluorous rhodamine as PS, cell viability was greatly reduced after illumination (Figure 16). For the application of this type of NEs, it is also important to prevent rapid leakage of PS. To this end, the authors increased the length of the lipophilic fluorinated alkyl group of the rhodamine derivative which resulted in a significant increase of the stability of this PS in NEs system. ${ }^{[246]}$ The obtained NEs formulation produced efficient phototoxic effect in cell culture according to NucGreen staining. Overall, with the development of dye-loaded NEs, PDT have a bright future for application as dual imaging and curing agent for cancer with minimal cytotoxic effects in the healthy tissues. 


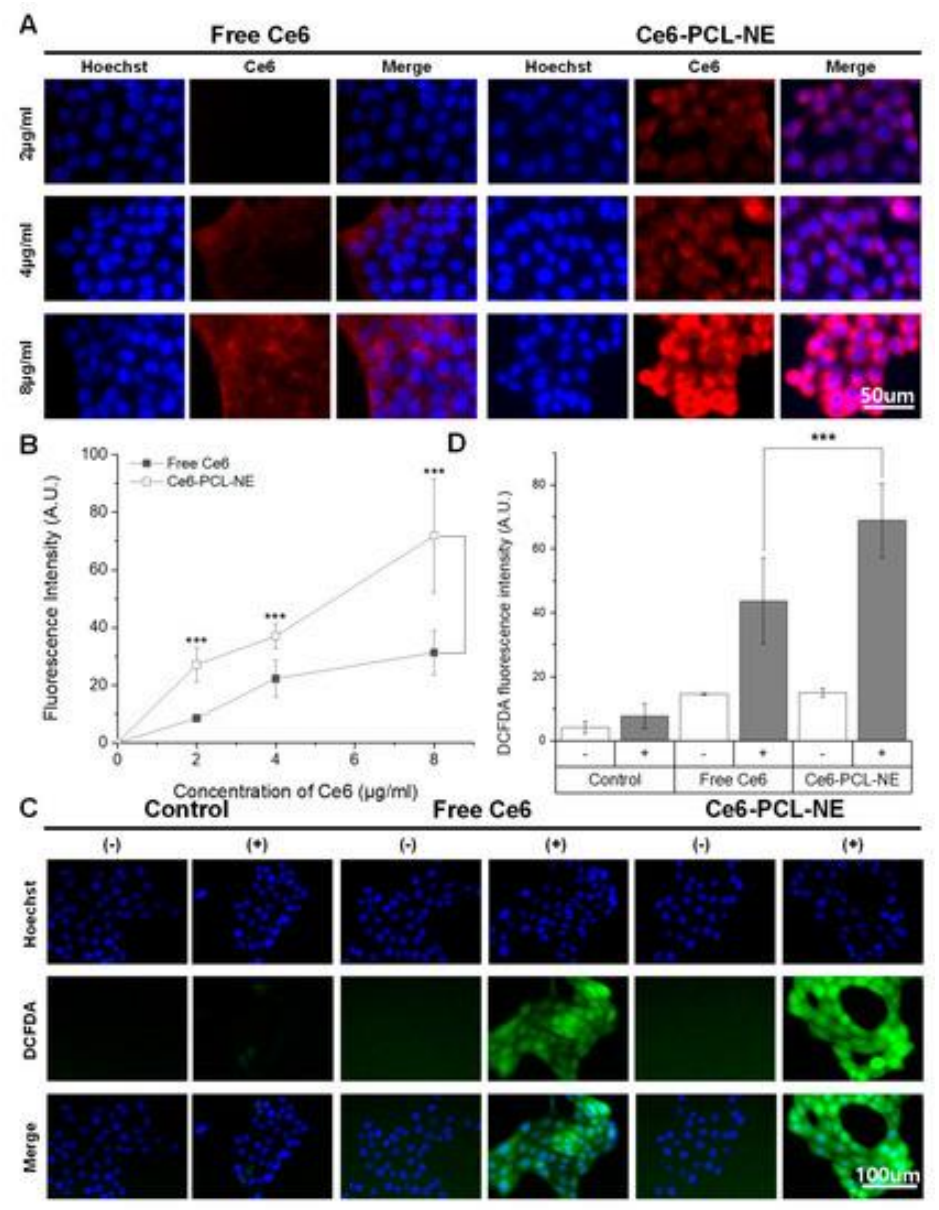

Figure 15. Cellular uptake of Ce6-PCL-NE and ROS generation. (A) Fluorescence images of 4T1 tumor cells treated by free Ce6 or Ce6-PCL-NE for 2 h. (B) Fluorescence intensity with different concentrations of Ce6. Results represent mean \pm s.d. $(\mathrm{n}=10)$. ${ }^{* * *} \mathrm{p}<0.001$. (C) Fluorescence signals of 2',7' -dichlorofluorescein diacetate (DCFDA) in 4T1 tumor cells treated with free Ce6 or Ce6-PCL-NE for $2 \mathrm{~h}$ with (+) or without (-) laser irradiation. (D) Fluorescence intensity of DCFDA in (C). *** $\mathrm{p}<0.001$. Results represent mean \pm s.d. (n = 10). Published with permission. ${ }^{[243]}$ Copyright 2019 Int. J. Mol. Sci.

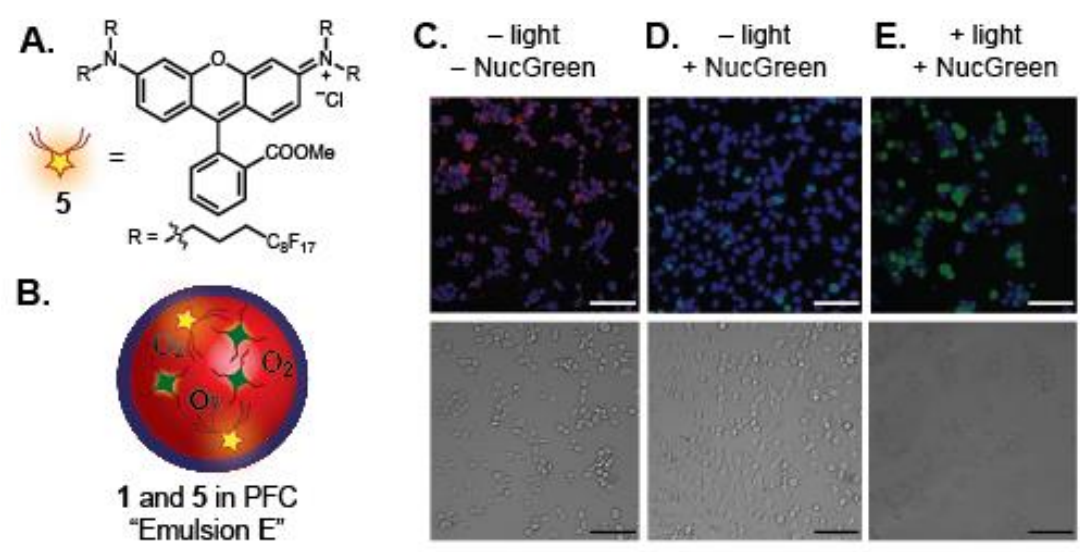

Figure 16. (A) Fluorous rhodamine 5. (B) PFC nanoemulsions containing rhodamine $5(0.57 \mathrm{mM})$ and fluorous photosensitizer $1(30 \mu \mathrm{M})$. (C/D/E) Confocal microscopy of HEK cells stained and incubated with emulsion $\mathrm{E}$ for $3 \mathrm{~h}$, washed, and stained with Hoescht dye. These cells were analyzed for rhodamine $(\mathrm{C}, \mathrm{Ex}$ $532 \mathrm{~nm}$ ), then subjected to the viability marker NucGreen ${ }^{\mathrm{TM}}$ and analyzed for cell death before (D, Ex 488) and after (E, Ex 488) light treatment $(420 \mathrm{~nm}, 8.5 \mathrm{~mW} / \mathrm{cm} 2,30 \mathrm{~min})$. Published with permission. ${ }^{[246]}$ Copyright 2018 Chem. Com. 


\section{Conclusion and perspectives}

Fluorescent nanoemulsions loaded with dyes is an expanding field for developing novel probes for biological and biomedical applications. It has a unique position with respect to all other existing nanoparticles, with its strong and weak points. On the side of advantages of NEs, one should mention its composition based on compounds generally recognized as safe, which place them together with liposomes, the golden standard in nanomedicine, ${ }^{[234]}$ and above all other systems in terms of biocompatibility and biodegradability. These "green" nanocarriers are mimic of lipoproteins and intracellular lipid droplets. Moreover, in contrast to most nanocarriers, it can be readily prepared in large quantities, making it comparable with industrial scale production ${ }^{[31]}$ Finally, in contrast to liposomes NEs have a large oil core, which can serve as a reservoir for encapsulating lipophilic drugs and contrast agents. Indeed, because of their oil core, NEs ensure the solubility and stability of lipophilic molecules. Actually, nanoemulsions are already frequently used as drug nanocarriers in some daily-used pharmaceutical products, such as topical products of drops or creams. ${ }^{[247,248]}$ Also, there already exist pediatric products in the form of nanoemulsions. ${ }^{[249]}$ Moreover, NEs constitute an important platform for future food industry. ${ }^{[250]}$ However, in the field of dye-loaded NEs a number of challenges should be addressed in order to establish them as efficient imaging agents in biology and medicine.

The present review identified these challenges and showed existing solutions. In particular, to obtain bright NPs, one should be able to encapsulate large amount of dyes without aggregation-caused quenching, which is a common tendency of flat $\pi$-conjugated fluorophores. ${ }^{[3]}$ Special dye design, which includes solubilizing lipophilic and bulky groups has been explored. Bulky counterions were particularly useful and they drastically increase solubility of cationic dyes, such as cyanines and prevent their aggregation through $\pi$ stacking. ${ }^{[12]}$ The second key issue is the dye leakage, which stems from the liquid nature of these particles. This issue is particularly difficult to address, because common methods including dialysis fail to estimate the leakage of lipophilic cargo poorly soluble in the medium. Ideally, the method should be compatible with biological media, such as serum, which serve as recipient medium for these lipophilic cargos. Therefore, advanced fluorescence techniques such as FRET and FCS has been applied to trace the fluorescent cargo in complex biological environments. ${ }^{[42,150]}$ Using these methods, it has been shown that dyes of intermediate lipophilicity, such as Nile Red, are not reliable fluorescent cargo for NEs, because in biological environment, they can rapidly leak out and localize in lipophilic binding sites, such as lipoproteins, biomembranes and intracellular lipid droplets. To prevent the leakage, dyes should be modified with long alkyl chains, as for instance in NR688 and cyanine derivatives, which ensures stability of dye-loaded NEs for hours.

Biological applications of NEs revealed their relatively low toxicity, probably because of GRAS components. Nevertheless, one should always take into account the toxicity and phototoxicity of dyes. Cellular studies showed remarkably low interactions of NEs with cell surface, which is clearly connected with the dense PEGylated surface made by a non-ionic surfactant. However, it is important to note that in vitro and in vivo imaging by NEs is frequently biased by dye leakage, especially commonly used Nile Red. It has been 
evidenced that on the time scale of 10-30 min, the dye can readily redistribute form NEs into cells or animal tissues, which can produce a wrong impression of NEs internalization. ${ }^{[42]}$ NEs loaded with lipophilic dyes resistant to leakage have shown to be remarkably stable in vivo in the blood with long circulation time in both zebrafish and mice. Remarkably, despite their liquid nature, dye-loaded NEs were able to enter tumors by the EPR effect in nearly intact form. ${ }^{[113]}$ Moreover, first examples of grafting specific ligands, such as RGD, showed possibilities of specific targeting. ${ }^{[172]}$ All these properties make NEs attractive as imaging agents to study EPR and specific targeting and as nanocarriers for drug delivery especially in the field of cancer. An important therapeutic modality that has already been demonstrated for dye-loaded NEs is photodynamic therapy. NEs can readily solubilize lipophilic photosensitizers in their oil core and decrease their accumulation in healthy tissues, thus can improve efficiency of PDT and minimize side effects.

Although those results showed the great potential of dye-loaded NEs as imaging and therapeutic agents, there still exist room for improvement. One aspect is that the size of NEs, which is relatively large (generally above $30 \mathrm{~nm}$ ) compared to proteins or some other nanoparticles. Ideally, it should be reduced to the range of 5-20 nm, which will be attractive for single particle tracking and super-resolution imaging, where the particle size should be comparable with biological macromolecules. ${ }^{[207,251]}$ This would require dramatic shift in the design strategy, for example with the use of more compact surfactants or combination of lipids with polymers. Another aspect is related to the improving of dye encapsulation, particle brightness and stability, which would require development of dyes with higher lipophilicity, fluorescence quantum yield and photostability as well as minimized selfquenching. In this respect, strategies based on bulky side groups ${ }^{[127]}$ and bulky hydrophobic counterions $^{[112,252]}$ should be further explored for known and new classes of fluorophores. ${ }^{[3]}$ A promising approach would be also to use aggregation-induced emission, which has been mainly applied so far for imaging intracellular lipid droplets. ${ }^{[122,253]}$ Last but not least, the surface chemistry and colloidal stability issues need to be addressed, because, non-specific interactions and off-target accumulation remains a major problem in nanomedicine. It is of great importance to continue working on new non-interacting hydrophilic shells for NEs in order to achieve high stability in biological media and long circulation time in vivo. Owing to stealth properties of NEs build of non-ionic surfactants, it is particularly attractive to covalently graft desired specific ligands to the NEs surface to enhance their specific targeting. However, the liquid nature of NEs makes this task particularly challenging, although the approaches based on modified lipids and amphiphilic polymers hold good promises. In this way, NEs can be used as nano-vehicles even for highly toxic drugs that can be delivered exclusively to the diseased tissues. Here, combination with dye-loaded NEs would enable theranostics, where the targeting capacity of the therapeutic NEs could be tracked for each patient. This is particularly important for precision medicine, where efficiency of a given nanomedicine relies greatly on section (stratification) of patients. ${ }^{[225]}$

Overall, owing to "green" biomimetic nature, simplicity of preparation, versatile of design, and stealth characteristics, dye-loaded NEs have the potential to become a universal tool for bioimaging, therapeutics and theranostics applications. 


\section{Acknowledgements}

This work was supported by the European Research Council ERC Consolidator grant BrightSens 648528. Fei LIU is supported from China scholarship council (CSC).

\section{Biographies}

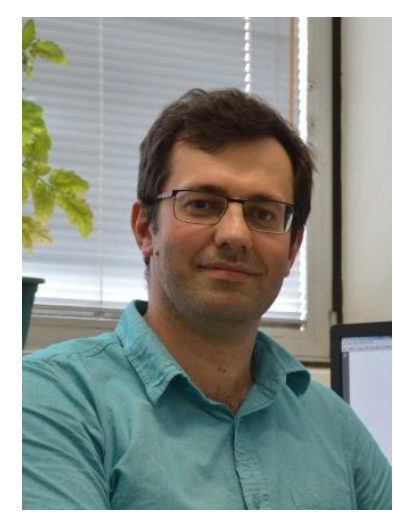

Andrey Klymchenko has received his PhD degree in 2003 from Kiev Taras Shevchenko University. After post-doctoral work at the University of Strasbourg and the Catholic University of Leuven, he joined CNRS at the University of Strasbourg in 2006. He got promoted to CNRS Research Director in 2014 and he is an ERC Consolidator fellow since 2015. He is a leader of "Nanochemistry and Bioimaging" group. He co-authors more than 200 peer-reviewed articles. His research interests include functional fluorescent molecules and nanomaterials for biosensing, imaging and theranostics.

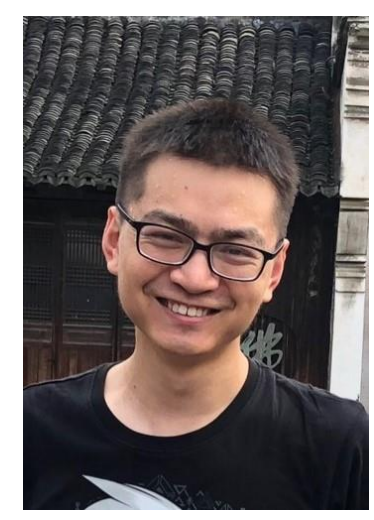

Fei Liu has received his Bachelor degree on package engineering in 2014 and Master degree of sugar engineering in 2017 from Tianjin university of science and technology. In 2018, Fei worked as Ph.D student at Paris institute of molecular chemistry in University Paris 6 on sugar chemistry. From 2019, Fei works at University of Strasbourg under the supervisor of Andrey Klymchenko as a Ph.D student. His research interests include covalent chemistry, functional nanoemulsions, fluorescent molecules for imaging, detection and theranostics. 


\section{References}

[1] M. I. J. Stich, L. H. Fischer, O. S. Wolfbeis, Chem. Soc. Rev. 2010, 39, 3102.

[2] P. D. Howes, R. Chandrawati, M. M. Stevens, Science. 2014, 346, 1247390.

[3] A. Reisch, A. S. Klymchenko, Small 2016, 12, 1968.

[4] D. Jin, P. Xi, B. Wang, L. Zhang, J. Enderlein, A. M. Van Oijen, Nat. Methods 2018, 15,415 .

[5] Y. Matsumoto, J. W. Nichols, K. Toh, T. Nomoto, H. Cabral, Y. Miura, R. J. Christie, N. Yamada, T. Ogura, M. R. Kano, et al., Nat. Nanotechnol. 2016, 11, 533.

[6] K. E. Sapsford, W. R. Algar, L. Berti, K. B. Gemmill, B. J. Casey, E. Oh, M. H. Stewart, I. L. Medintz, Chem. Rev. 2013, 113, 1904.

[7] A. Heuer-Jungemann, N. Feliu, I. Bakaimi, M. Hamaly, A. Alkilany, I. Chakraborty, A. Masood, M. F. Casula, A. Kostopoulou, E. Oh, et al., Chem. Rev. 2019, 119, 4819.

[8] X. Michalet, F. F. Pinaud, L. A. Bentolila, J. M. Tsay, S. Doose, J. J. Li, G. Sundaresan, A. M. Wu, S. S. Gambhir, S. Weiss, Science. 2005, 307, 538.

[9] M. Bruchez, M. Moronne, P. Gin, S. Weiss, A. P. Alivisatos, Science. 1998, 281, 2013.

[10] S. W. Bae, W. Tan, J. I. Hong, Chem. Commun. 2012, 48, 2270.

[11] M. Montalti, L. Prodi, E. Rampazzo, N. Zaccheroni, Chem. Soc. Rev. 2014, 43, 4243.

[12] M. Haase, H. Schäfer, Angew. Chemie - Int. Ed. 2011, 50, 5808.

[13] S. Wen, J. Zhou, K. Zheng, A. Bednarkiewicz, X. Liu, D. Jin, Nat. Commun. 2018, 9, 2415 .

[14] O. Zavoiura, U. Resch-Genger, O. Seitz, Bioconjug. Chem. 2018, 29, 1690.

[15] G. Xu, S. Zeng, B. Zhang, M. T. Swihart, K.-T. Yong, P. N. Prasad, Chem. Rev. 2016, $116,12234$.

[16] P. Frantsuzov, M. Kuno, B. Jankó, R. A. Marcus, Nat. Phys. 2008, 4, 519.

[17] H. Li, C. Wang, Z. Peng, X. Fu, J. Nanomater. 2015, 2015, ID 519385.

[18] A. Gnach, A. Bednarkiewicz, Nano Today 2012, 7, 532.

[19] H.-S. Peng, D. T. Chiu, Chem. Soc. Rev. 2015, 44, 4699.

[20] C. Wu, D. T. Chiu, Angew. Chemie - Int. Ed. 2013, 52, 3086.

[21] J. Li, K. Pu, Chem. Soc. Rev. 2019, 48, 38.

[22] K. Li, B. Liu, Chem. Soc. Rev. 2014, 43, 6570.

[23] A. Vollrath, S. Schubert, U. S. Schubert, J. Mater. Chem. B 2013, 1, 1994.

[24] A. T. Gomez, C. Zeppek, H. Amenitsch, F. Uhlig, 2016.

[25] P. Yingchoncharoen, D. S. Kalinowski, D. R. Richardson, Pharmacol. Rev. 2016, 68, 701. 
[26] Z. Yaari, D. Da Silva, A. Zinger, E. Goldman, A. Kajal, R. Tshuva, E. Barak, N. Dahan, D. Hershkovitz, M. Goldfeder, et al., Nat. Commun. 2016, 7, 1.

[27] W. Mehnert, K. Mäder, Adv. Drug Deliv. Rev. 2001, 47, 165.

[28] S. Nasirizadeh, B. Malaekeh-Nikouei, J. Drug Deliv. Sci. Technol. 2020, 55, 101458.

[29] R. H. Müller, K. Mäder, S. Gohla, Eur. J. Pharm. Biopharm. 2000, 50, 161.

[30] R. V. Tikekar, N. Nitin, Soft Matter 2011, 7, 8149.

[31] S. M. Jafari, D. J. McClements, Nanoemulsions : Formulation, Applications, and Characterization. 2018.

[32] N. Anton, T. F. Vandamme, Pharm. Res. 2011, 28, 978.

[33] Y. Singh, J. Gopal Meher, K. Raval, A. Khan, M. Chaurasia, N. K. Jain, M. K. Chourasia, J.Control.Release. 2017, 252, 28

[34] S. Akram, X. Wang, T. F. Vandamme, M. Collot, A. U. Rehman, N. Messaddeq, Y. Mély, N. Anton, Langmuir 2019, 35, 2313.

[35] T. Sheth, S. Seshadri, T. Prileszky, M. E. Helgeson, Nat. Rev. Mater. 2020, 5, 214.

[36] N. Anton, T. F. Vandamme, Int. J. Pharm. 2009, 377, 142.

[37] D. J. McClements, Soft Matter 2012, 8, 1719.

[38] M. Yao, H. Xiao, D. J. McClements, Annu. Rev. Food Sci. Technol. 2014, 5, 53.

[39] M. Kumar, R. S. Bishnoi, A. K. Shukla, C. P. Jain, Prev. Nutr. Food Sci. 2019, 24, 225 .

[40] C. Lovelyn, A. A. Attama, J. Biomater. Nanobiotechnol. 2011, 02, 626.

[41] K. Pathak, S. Pattnaik, K. Swain, in Nanoemulsions Formul. Appl. Charact., Elsevier Inc., 2018, pp. 415-433.

[42] A. S. Klymchenko, E. Roger, N. Anton, H. Anton, I. Shulov, J. Vermot, Y. Mely, T. F. Vandamme, RSC Adv. 2012, 2, 11876.

[43] X. Li, N. Anton, G. Zuber, Adv. Drug Deliv. Rev. 2014, 76, 116.

[44] N. Anton, F. Hallouard, M. F. Attia, T. F. Vandamme, Springer, Cham, 2016, pp. 273300 .

[45] A. Prokop, V. Weissig. 2016, Intracellular Delivery III.

[46] X. Li, N. Anton, G. Zuber, M. Zhao, N. Messaddeq, F. Hallouard, H. Fessi, T. F. Vandamme, Biomaterials 2013, 34, 481.

[47] S. Martin, R. G. Parton, Nat. Rev. Mol. Cell Biol. 2006, 7, 373.

[48] Y. Guo, K. R. Cordes, R. V Farese, T. C. Walther, J. Cell Sci. 2009, 122, 749.

[49] D. Sobot, S. Mura, S. O. Yesylevskyy, L. Dalbin, F. Cayre, G. Bort, J. Mougin, Di. Desmaële, S. Lepetre-Mouelhi, G. Pieters, et al., Nat. Commun. 2017, 8, 15678.

[50] K. K. Ng, J. F. Lovell, G. Zheng, Acc. Chem. Res. 2011, 44, 1105. 
[51] Y.-Z. Du, S.-N. Li, Y.-L. Feng, D.-L. Yu, F.-Q. Hu, H. Yuan, S. He, S. P. Jiang, X.-Y. Lu, Int. J. Nanomedicine 2013, 8, 3141.

[52] S. Anuchapreeda, Y. Fukumori, S. Okonogi, H. Ichikawa, J. Nanotechnol. 2012, 2012, 1 .

[53] Y. Y. Hwang, K. Ramalingam, D. R. Bienek, V. Lee, T. You, R. Alvarez, Antimicrob. Agents Chemother. 2013, 57, 3568.

[54] V. Ghosh, A. Mukherjee, N. Chandrasekaran, Colloids Surfaces B Biointerfaces 2014, $114,392$.

[55] K. A. Abd-Elsalam, A. R. Khokhlov, Appl. Nanosci. 2015, 5, 255.

[56] T. K. Vyas, A. Shahiwala, M. M. Amiji, Int. J. Pharm. 2008, 347, 93.

[57] S. Al-Edresi, S. Baie, Int. J. Pharm. 2009, 373, 174.

[58] D. S. Bernardi, T. A. Pereira, N. R. Maciel, J. Bortoloto, G. S. Viera, G. C. Oliveira, P. A. Rocha-Filho, J. Nanobiotechnology 2011, 9, 44.

[59] D. Bera, D. Lahiri, A. Nag, J. Food Eng. 2006, 74, 542.

[60] V. K. Pawar, S. B. Panchal, Y. Singh, J. G. Meher, K. Sharma, P. Singh, H. K. Bora, A. Singh, D. Datta, M. K. Chourasia, J. Control. Release 2014, 196, 295.

[61] B. Ozturk, S. Argin, M. Ozilgen, D. J. McClements, Food Chem. 2015, 188, 256.

[62] J. M. Morais Diane, J. Burgess, Int. J. Pharm. 2014, 465, 455.

[63] X. Wang, N. Anton, P. Ashokkumar, H. Anton, T. K. Fam, T. Vandamme, A. S. Klymchenko, M. Collot, ACS Appl. Mater. Interfaces 2019, 11, 13079.

[64] M. S. Ali, M. S. Alam, N. Alam, M. R. Siddiqui, Iran. J. Pharm. Res. IJPR 2014, 13, 1125 .

[65] B. Sharif Makhmalzadeh, S. Torabi, A. Azarpanah, Iran. J. Pharm. Res. IJPR 2012, $11,47$.

[66] X. Wang, Y. Jiang, Y.-W. Wang, M.-T. Huang, C.-T. Ho, Q. Huang, Food Chem. 2008, 108, 419.

[67] A. Azeem, M. Rizwan, F. J. Ahmad, Z. Iqbal, R. K. Khar, M. Aqil, S. Talegaonkar, AAPS PharmSciTech 2009, 10, 69.

[68] Y.-J. Jo, Y.-J. Kwon, Food Sci. Biotechnol. 2014, 23, 107.

[69] V. Polychniatou, C. Tzia, J. Am. Oil Chem. Soc. 2014, 91, 79.

[70] C. Jadhav, V. Kate, S. A. Payghan, J. Nanostructure Chem. 2015, 5, 107.

[71] K. J. Scheller, S. J. Williams, A. J. Lawrence, B. Jarrott, E. Djouma, MethodsX 2014, $1,212$.

[72] C. Brüsewitz, A. Schendler, A. Funke, T. Wagner, R. Lipp, Int. J. Pharm. 2007, 329, 173.

[73] W. Wu, W. He, Y. Tan, Z. Tian, L. Chen, F.-Q. Hu, W. Wu, Int. J. Nanomedicine 2011, 6, 521. 
[74] P. G. Maher, M. A. Fenelon, Y. Zhou, M. Kamrul Haque, Y. H. Roos, J. Food Sci. 2011, 76, C1108.

[75] A. Ali, G. Mekhloufi, N. Huang, F. Agnely, Int. J. Pharm. 2016, 500, 291.

[76] J. Combrinck, A. Otto, J. du Plessis, AAPS PharmSciTech 2014, 15, 588.

[77] N. Rapoport, D. A. Christensen, A. M. Kennedy, K.-H. Nam, Ultrasound Med. Biol. 2010, 36, 419 .

[78] V. V. Erramreddy, S. Ghosh, Langmuir 2014, 30, 11062.

[79] S. Uluata, E. A. Decker, D. J. McClements, Food Biophys. 2016, 11, 52.

[80] M. Kanamaru, Y. Einaga, Polymer (Guildf). 2002, 43, 3925.

[81] S. Hirsjärvi, S. Dufort, J. Gravier, I. Texier, Q. Yan, J. Bibette, L. Sancey, V. Josserand, C. Passirani, J. P. Benoit, et al., Nanomedicine Nanotechnology, Biol. Med. 2013, 9, 375.

[82] N. T. Huynh, C. Passirani, P. Saulnier, J. P. Benoit, Int. J. Pharm. 2009, 379, 201.

[83] M. Adamczak, G. Para, C. Simon, P. Warszyński, J. Microencapsul. 2013, 30, 479.

[84] J. Zhuang, M. Ying, K. Spiekermann, M. Holay, Y. Zhang, F. Chen, H. Gong, J. H. Lee, W. Gao, R. H. Fang, et al., Adv. Mater. 2018, 30, 1804693.

[85] M. F. Attia, S. M. Dieng, M. Collot, A. S. Klymchenko, C. Bouillot, C. A. Serra, M. Schmutz, M. Er-Rafik, T. F. Vandamme, N. Anton, Macromol. Biosci. 2017, 17, 1600471.

[86] R. Vecchione, G. Iaccarino, P. Bianchini, R. Marotta, F. D'autilia, V. Quagliariello, A. Diaspro, P. A. Netti, Small 2016, 12, 3005.

[87] N. Anton, T. F. Vandamme, in Handb. Nanoparticles, Springer International Publishing, Cham, 2015, pp. 93-116.

[88] K. S. Pafiti, E. Loizou, C. S. Patrickios, L. Porcar, Macromolecules 2010, 43, 5195.

[89] T. Tadros, P. Izquierdo, J. Esquena, C. Solans, Adv. Colloid Interface Sci. 2004, 108109, 303.

[90] N. Anton, S. Akram, T. F. Vandamme, in Nanoemulsions Formul. Appl. Charact., Elsevier Inc., 2018, pp. 77-100.

[91] S. Aidarova, A. Sharipova, J. Krägel, R. Miller, Adv Colloid Interfac, 2014, 205, 87.

[92] K. Szczepanowicz, U. Bazylińska, J. Pietkiewicz, L. Szyk-Warszyńska, K. A. Wilk, P. Warszyński, Adv Colloid Interfac. 2014, 222, 678.

[93] T. Nylander, Y. Samoshina, B. Lindman, Adv Colloid Interfac. 2006, 123, 105.

[94] A. Sharipova, S. Aidarova, P. Cernoch, R. Miller, Physicochem. Eng. Asp. 2013, 438, 141.

[95] Á. Ábrahám, A. Kardos, A. Mezei, R. A. Campbell, I. Varga, Langmuir 2014, 30, 4970. 
[96] C. D. Bain, P. M. Claesson, D. Langevin, R. Meszaros, T. Nylander, C. Stubenrauch, S. Titmuss, R. Von Klitzing, Adv. Colloid Interface Sci. 2010, 155, 32.

[97] G. B. Sukhorukov, E. Donath, H. Lichtenfeld, E. Knippel, M. Knippel, A. Budde, H. Möhwald, Colloids Surfaces A Physicochem. Eng. Asp. 1998, 137, 253.

[98] K. Ariga, Q. Ji, J. P. Hill, A. Vinu, Soft Matter 2009, 5, 3562.

[99] F. Quemeneur, M. Rinaudo, G. Maret, B. Pépin-Donat, Soft Matter 2010, 6, 4471.

[100] H. A. Jerri, R. A. Dutter, D. Velegol, Soft Matter 2009, 5, 827.

[101] $*, \dagger, \dagger$ Xin Rong Teng, $*, \dagger$ and Dmitry G. Shchukin, H. Möhwald $\dagger$, Langmuir 2008, 24 (2), 383-389

[102] C. Preetz, A. Rübe, I. Reiche, G. Hause, K. Mäder, Nanomedicine Nanotechnology, Biol. Med. 2008, 4, 106.

[103] K. Szczepanowicz, D. Dronka-Góra, G. Para, P. Warszyński, J. Microencapsul. 2010, 27, 198.

[104] D. Guzey, D. J. McClements, Adv. Colloid Interface Sci. 2006, 128-130, 227.

[105] U. Bazyli, R. Skrzela, K. Szczepanowicz, P. Warszy, K. A. Wilk, Soft Matter. 2011, 7, 6113

[106] K. Szczepanowicz, H. J. Hoel, L. Szyk-Warszynska, E. Bielańska, A. M. Bouzga, G. Gaudernack, C. Simon, P. Warszynski, Langmuir 2010, 26, 12592.

[107] D. J. F. Taylor, R. K. Thomas, J. Penfold, Curr. Opin. Colloid Interface Sci. 2007, $11(6), 337$.

[108] $*, \dagger$ D. O. Grigoriev, $\ddagger$ T. Bukreeva, $\dagger$ and H. Möhwald, D. G. Shchukin $\dagger$, Langmuir 2008, 24(3), 999-1004.

[109] L. Nilsson, B. Bergenståhl, J. Colloid Interface Sci. 2007, 308, 508.

[110] W. Men, P. Zhu, S. Dong, W. Liu, K. Zhou, Y. Bai, X. Liu, S. Gong, S. Zhang, Drug Deliv. 2020, 27, 180.

[111] H. Zhou, Y. Yue, G. Liu, Y. Li, J. Zhang, Q. Gong, Z. Yan, M. Duan, Nanoscale Res. Lett. 2010, 5, 224.

[112] V. N. Kilin, H. Anton, N. Anton, E. Steed, J. Vermot, T. F. Vandamme, Y. Mely, A. S. Klymchenko, Biomaterials 2014, 35, 4950.

[113] R. Bouchaala, L. Mercier, B. Andreiuk, Y. Mély, T. Vandamme, N. Anton, J. G. Goetz, A. S. Klymchenko, J. Control. Release 2016, 236, 57.

[114] I. Texier, M. Goutayer, A. Da Silva, L. Guyon, N. Djaker, V. Josserand, E. Neumann, J. Bibette, F. Vinet, J. Biomed. Opt. 2009, 14, 054005.

[115] P. A. Jarzyna, T. Skajaa, A. Gianella, D. P. Cormode, D. D. Samber, S. D. Dickson, W. Chen, A. W. Griffioen, Z. A. Fayad, W. J. M. Mulder, Biomaterials 2009, 30, 6947.

[116] A. Jacquart, M. Kéramidas, J. Vollaire, R. Boisgard, G. Pottier, E. Rustique, F. Mittler, F. P. Navarro, J. Boutet, J.-L. Coll, et al., J. Biomed. Opt. 2013, 18, 101311. 
[117] A. Gianella, P. A. Jarzyna, V. Mani, S. Ramachandran, C. Calcagno, J. Tang, B. Kann, W. J. R. Dijk, V. L. Thijssen, A. W. Griffioen, et al., ACS Nano. 2011, 5(6), 4422.

[118] J. Gravier, F. P. Navarro, T. Delmas, F. Mittler, A.-C. Couffin, F. Vinet, I. Texier, J. Biomed. Opt. 2011, 16, 096013.

[119] J. A. Rochira, M. V. Gudheti, T. J. Gould, R. R. Laughlin, J. L. Nadeau, S. T. Hess, J. Phys. Chem. C 2007, 111, 1695.

[120] D. R. Larson, H. Ow, H. D. Vishwasrao, A. A. Heikal, U. Wiesner, W. W. Webb, Chem. Mater. 2008, 20, 2677.

[121] G. Sun, M. Y. Berezin, J. Fan, H. Lee, J. Ma, K. Zhang, K. L. Wooley, S. Achilefu, Nanoscale 2010, 2, 548.

[122] J. Mei, N. L. C. Leung, R. T. K. Kwok, J. W. Y. Lam, B. Z. Tang, Chem. Rev. 2015, $115,11718$.

[123] S. Chen, H. Wang, Y. Hong, B. Z. Tang, Mater. Horizons 2016, 3, 283.

[124] L. Yan, Y. Zhang, B. Xu, W. Tian, Nanoscale 2016, 8, 2471.

[125] T. Heek, C. Fasting, C. Rest, X. Zhang, F. Würthner, R. Haag, Chem. Commun. 2010, $46,1884$.

[126] D. Li, C. L. Schreiber, B. D. Smith, Angew. Chemie Int. Ed. 2020, 59(29), 12154.

[127] B. Zhang, H. Soleimaninejad, D. J. Jones, J. M. White, K. P. Ghiggino, T. A. Smith, W. W. H. Wong, Chem. Mater. 2017, 29, 8395.

[128] K. Trofymchuk, A. Reisch, I. Shulov, Y. Mély, A. S. Klymchenko, Nanoscale 2014, 6, 12934.

[129] F. Würthner, T. E. Kaiser, C. R. Saha-Möller, Angew. Chemie Int. Ed. 2011, 50, 3376.

[130] A. Reisch, P. Didier, L. Richert, S. Oncul, Y. Arntz, Y. Mély, A. S. Klymchenko, Nat. Commun. 2014, 5, 4089.

[131] L. D. Lavis, R. T. Raines, ACS Chem. Biol. 2008, 3, 142.

[132] K. Zyabrev, A. Doroshenko, E. Mikitenko, Y. Slominskii, A. Tolmachev, European J. Org. Chem. 2008, 2008, 1550.

[133] A. O. Gerasov, M. P. Shandura, Y. P. Kovtun, Dye. Pigment. 2008, 77, 598.

[134] A. O. Gerasov, M. P. Shandura, Y. P. Kovtun, Dye. Pigment. 2008, 79, 252.

[135] A. O. Gerasov, K. V. Zyabrev, M. P. Shandura, Y. P. Kovtun, Dye. Pigment. 2011, 89, 76.

[136] M. Collot, † Tkhe, K. Fam, P. Ashokkumar, O. Faklaris, T. Galli, L. Danglot, A. S. Klymchenko, J. Am. Chem. Soc. 2018, 140(16), 5401-5411.

[137] M. K. Kuimova, Phys. Chem. Chem. Phys. 2012, 14, 12671.

[138] M. A. Haidekker, E. A. Theodorakis, Org. Biomol. Chem. 2007, 5, 1669.

[139] J. M. Janjic, P. Shao, S. Zhang, X. Yang, S. K. Patel, M. Bai, Biomaterials, 2014, $35(18), 4958$. 
[140] U. Bazylin’ska, B. Bazylin’ska, J. Pietkiewicz, J. Saczko, M. Nattich-Rak, J. Rossowska, A. Garbiec, K. A. Wilk. Eur J Pharm Sci, 2012,47(2),406.

[141] K. Kassab, J. Photochem. Photobiol. B Biol. 2002, 68, 15.

[142] R. Bouchaala, N. Anton, H. Anton, T. Vandamme, J. Vermot, D. Smail, Y. Mély, A. S. Klymchenko, Colloids Surfaces B Biointerfaces 2017, 156, 414.

[143] S. Snipstad, S. Hak, H. Baghirov, E. Sulheim, Ý. Mørch, S. Lélu, E. von Haartman, M. Bäck, K. P. R. Nilsson, A. S. Klymchenko, et al., Cytom. Part A 2017, 91, 760.

[144] Q. Wang, R. Choy, Q. Dai, A. E. Ostafin, J. Non. Cryst. Solids 2007, 353, 354.

[145] A. Wagh, S. Y. Qian, B. Law, Bioconjug. Chem. 2012, 23, 981.

[146] D. J. McClements, Y. Li, Adv. Colloid Interface Sci. 2010, 159, 213.

[147] K. Ahmed, Y. Li, D. J. McClements, H. Xiao, Food Chem. 2012, 132, 799.

[148] E. M. Sletten, T. M. Swager, J. Am. Chem. Soc. 2014, 136, 13574.

[149] S. A. Abouelmagd, B. Sun, A. C. Chang, Y. J. Ku, Y. Yeo, Mol. Pharm. 2015, 12, 997.

[150] R. Bouchaala, L. Richert, N. Anton, T. F. Vandamme, S. Djabi, Y. Meíy, A. S. Klymchenko, ACS Omega. 2018, 3(10) 14333.

[151] U. Bazylińska, R. Skrzela, K. Szczepanowicz, P. Warszyński, K. A. Wilk, Soft Matter 2011, 7, 6113.

[152] Y. Qiu, H. Hu, D. Zhao, J. Wang, H. Wang, Q. Wang, H. Peng, Y. Liao, X. Xie, Polymer. 2019, 170, 7.

[153] S. Bhargava, J. J. H. Chu, S. Valiyaveettil, ACS Omega 2018, 3, 7663.

[154] Y. Wen, W. Zhang, X. Zhu, J. Zhang, L. Wang, Energy 2018, 158, 537.

[155] S. W. Morton, X. Zhao, M. A. Quadir, P. T. Hammond, Biomaterials 2014, 35, 3489.

[156] Y. Zhao, I. Van Rooy, S. Hak, F. Fay, J. Tang, C. D. L. Davies, M. Skobe, E. A. Fisher, A. Radu, Z. A. Fayad, et al., ACS Nano 2013, 7, 10362.

[157] J. Gravier, L. Sancey, S. Hirsjärvi, E. Rustique, C. Passirani, J. P. Benoît, J. L. Coll, I. Texier, Mol. Pharm. 2014, 11, 3133.

[158] B. T. Bajar, E. S. Wang, S. Zhang, M. Z. Lin, J. Chu, Sensors (Switzerland) 2016, 16, 1.

[159] K. E. Sapsford, L. Berti, I. L. Medintz, Angew. Chemie Int. Ed. 2006, 45(28), 4562.

[160] E. L. Elson, Biophys. J. 2011, 101, 2855.

[161] D. Wöll, RSC Adv. 2014, 4, 2447.

[162] T. Liedl, S. Keller, F. C. Simmel, J. O. Rädler, W. J. Parak, Small 2005, 1, 997.

[163] C. Simonsson, G. Bastiat, M. Pitorre, A. S. Klymchenko, J. Béjaud, Y. Mély, J.-P. Benoit, Eur. J. Pharm. Biopharm. 2016, 98, 47. 
[164] B. L. Sanchez-Gaytan, F. Fay, S. Hak, A. Alaarg, Z. A. Fayad, C. Pérez-Medina, W. J. M Mulder, Y. Zhao, Angew Chem Int Ed Engl 2017, 56, 2923.

[165] a. L. Lainé, J. Gravier, M. Henry, L. Sancey, J. Béjaud, E. Pancani, M. Wiber, I. Texier, J. L. Coll, J. P. Benoit, et al., J. Control. Release 2014, 188, 1.

[166] E. Roger, J. C. Gimel, C. Bensley, A. S. Klymchenko, J. P. Benoit, J. Control. Release 2017, 253, 11 .

[167] X. Wang, M. Collot, Z. Omran, T. F. Vandamme, A. Klymchenko, N. Anton, J. Colloid Interface Sci. 2020, 578, 768.

[168] S. Bou, X. Wang, N. Anton, A. S. Klymchenko, M. Collot, J. Mater. Chem. B, 2020, $8,5938-5944$.

[169] S. K. Patel, M. J. Patrick, J. A. Pollock, J. M. Janjic, J. Biomed. Opt. 2013, 18, 101312.

[170] E.-H. Lee, J.-K. Kim, J.-S. Lim, S.-J. Lim, Colloids Surfaces B Biointerfaces 2015, $136,305$.

[171] H. Z. An, M. E. Helgeson, P. S. Doyle, Adv. Mater. 2012, 24, 3838.

[172] M. Goutayer, S. Dufort, V. Josserand, A. Royère, E. Heinrich, F. Vinet, J. Bibette, J. L. Coll, I. Texier, Eur. J. Pharm. Biopharm. 2010, 75, 137.

[173] J. Choi, E. Rustique, M. Henry, M. Guidetti, V. Josserand, L. Sancey, J. Boutet, J.-L. Coll, Int. J. Pharm. 2017, 532, 677.

[174] A. Saito, S. Yamamoto, R. Ochi, K. Inoue, S. Hadano, S. Watanabe, T. Nakayama, Y. Niko, Bull. Chem. Soc. Jpn. 2020, 93, 568.

[175] M. Attia, M. I. Swasy, R. Akasov, F. Alexis, D. C. Whitehead, ACS Appl. Bio Mater. 2020, DOI.org/10.1021/acsabm.0c00567.

[176] M. F. Attia, S. M. Dieng, M. Collot, A. S. Klymchenko, C. Bouillot, C. A. Serra, M. Schmutz, M. Er-Rafik, T. F. Vandamme, N. Anton, Macromol. Biosci. 2017, 17, 1600471 .

[177] S. Bou, X. Wang, N. Anton, R. Bouchaala, A. S. Klymchenko, M. Collot, Soft Matter 2020, 16, 4173.

[178] V. Calcagno, R. Vecchione, V. Quagliariello, P. Marzola, A. Busato, P. Giustetto, M. Profeta, S. Gargiulo, C. Di Cicco, H. Yu, et al., Adv. Healthc. Mater. 2019, 8, 1801313.

[179] G. Sahay, D. Y. Alakhova, A. V. Kabanov, J. Control. Release 2010, 145, 182.

[180] A. Vollrath, A. Schallon, C. Pietsch, S. Schubert, T. Nomoto, Y. Matsumoto, K. Kataoka, U. S. Schubert, Soft Matter 2013, 9, 99.

[181] S. Patel, J. Kim, M. Herrera, A. Mukherjee, A. V Kabanov, G. Sahay, Adv. Drug Deliv. Rev. 2019, 144, 90

[182] S. D. Conner, S. L. Schmid, Nature 2003, 422, 37.

[183] W. Jiang, B. Y. S. Kim, J. T. Rutka, W. C. W. Chan, Nat. Nanotechnol. 2008, 3, 145.

[184] M. Yao, L. He, D. J. McClements, H. Xiao, J. Agric. Food Chem. 2015, 63, 8044. 
[185] J. Zheng, Y. Li, M. Song, X. Fang, Y. Cao, D. J. McClements, H. Xiao, Food Res. Int. 2014, 62, 98.

[186] O. Harush-Frenkel, E. Rozentur, S. Benita, Y. Altschuler, Biomacromolecules 2008, 9, 435.

[187] Y. Luo, Z. Teng, T. T. Y. Wang, Q. Wang, J. Agric. Food Chem. 2013, 61, 7621.

[188] H. S. Ribeiro, J. M. M. Guerrero, K. Briviba, G. Rechkemmer, H. P. Schuchmann, H. Schubert, J. Agric. Food Chem. 2006, 54, 9366.

[189] Y. Fan, Y. Zhang, W. Yokoyama, J. Yi, Nanomaterials 2017, 7, 349.

[190] J. Yi, Y. Li, F. Zhong, W. Yokoyama, Food Hydrocoll. 2014, 35, 19.

[191] A. C. Anselmo, M. Zhang, S. Kumar, D. R. Vogus, S. Menegatti, M. E. Helgeson, S. Mitragotri, ACS Nano 2015, 9, 3169.

[192] X. Yi, X. Shi, H. Gao, Phys. Rev. Lett. 2011, 107, 098101.

[193] M. Herant, V. Heinrich, M. Dembo, J. Cell Sci. 2005, 118, 1789.

[194] M. D. Howard, M. Jay, T. D. Dziubla, X. Lu, J. Biomed. Nanotechnol. 2008, 4, 133.

[195] J. V. Jokerst, T. Lobovkina, R. N. Zare, S. S. Gambhir, Nanomedicine 2011, 6, 715.

[196] S. Hak, E. Helgesen, H. H. Hektoen, E. M. Huuse, P. A. Jarzyna, W. J. M. Mulder, O. Haraldseth, C. D. L. Davies, ACS Nano 2012, 6, 5648.

[197] M. Coelho, N. Maghelli, I. M. Tolić-Nørrelykke, Integr. Biol. 2013, 5, 748.

[198] M. Pawlicki, H. A. Collins, R. G. Denning, H. L. Anderson, Angew. Chemie - Int. Ed. 2009, 48, 3244.

[199] P. G. Bush, D. L. Wokosin, A. C. Hall, Front. Biosci. 2007, 12, 2646.

[200] H. M. Kim, B. R. Cho, Chem. Rev. 2015, 115, 5014.

[201] S. Hou, C. Johnson, K. Welsher, Molecules 2019, 24, 2826.

[202] M. Dahan, S. Lévi, C. Luccardini, P. Rostaing, B. Riveau, A. Triller, Science (80-. ). 2003, 302, 442 .

[203] Y. Wang, R. Hu, G. Lin, I. Roy, K.-T. Yong, ACS Appl. Mater. Interfaces 2013, 5, 2786.

[204] M. U. Zahid, L. Ma, S. J. Lim, A. M. Smith, Nat. Commun. 2018, 9, 1.

[205] E. Genin, Z. Gao, J. A. Varela, J. Daniel, T. Bsaibess, I. Gosse, L. Groc, L. Cognet, M. Blanchard-Desce, Adv. Mater. 2014, 26, 2258.

[206] J. Yu, C. Wu, S. P. Sahu, L. P. Fernando, C. Szymanski, J. McNeill, J. Am. Chem. Soc. 2009, 131, 18410.

[207] A. Reisch, D. Heimburger, P. Ernst, A. Runser, P. Didier, D. Dujardin, A. S. Klymchenko, Adv. Funct. Mater. 2018, 28, 1805157.

[208] B. Arechabala C. Coiffard P. Rivalland L. J. M. Coiffard Y. De Roeck - Holtzhauer, J. Appl. Toxicol. 1999, 19(3), 163. 
[209] H. Yoon, X. Zhang, M. Kang, G. Kim, S. Shin, S. Baek, B. Lee, S. Hong, J. Kim, K. Hong, et al., Int. J. Mol. Sci. 2018, 19, 280.

[210] C. Vater, A. Adamovic, L. Ruttensteiner, K. Steiner, P. Tajpara, V. Klang, A. ElbeBürger, M. Wirth, C. Valenta, Int. J. Pharm. 2019, 566, 383.

[211] D. J. Stephens, V. J. Allan, Science 2003, 300, 82.

[212] A. Khodjakov, C. L. Rieder, Methods 2006, 38, 2.

[213] P. P. Laissue, R. A. Alghamdi, P. Tomancak, E. G. Reynaud, H. Shroff, Nat. Methods 2017, 14, 657 .

[214] S. Luo, E. Zhang, Y. Su, T. Cheng, C. Shi, Biomaterials 2011, 32, 7127.

[215] V. J. Pansare, S. Hejazi, W. J. Faenza, R. K. Prud'Homme, Chem. Mater. 2012, 24, 812.

[216] S. K. Ko, X. Chen, J. Yoon, I. Shin, Chem. Soc. Rev. 2011, 40, 2120.

[217] V. Hyenne, S. Ghoroghi, M. Collot, J. Bons, G. Follain, S. Harlepp, B. Mary, J. Bauer, L. Mercier, I. Busnelli, et al., Dev. Cell 2019, 48, 554.

[218] F. J. Verweij, V. Hyenne, G. Van Niel, J. G. Goetz, Trends Cell Biol. 2019, 29, 770.

[219] B. S. Schuster, L. M. Ensign, D. B. Allan, J. S. Suk, J. Hanes, Adv. Drug Deliv. Rev. $\mathbf{2 0 1 5}, 91,70$.

[220] S. K. Lyons, Cancer J. (United States) 2015, 21, 152.

[221] L. Yuan, W. Lin, K. Zheng, L. He, W. Huang, Chem. Soc. Rev 2013, 42, 622.

[222] S. A. Hilderbrand, R. Weissleder, Curr Opin Chem Biol. 2010, 14(1), 71.

[223] H. Kobayashi, M. Ogawa, R. Alford, P. L. Choyke, Y. Urano, Chem. Rev. 2010, 110, 2620.

[224] Z. Guo, S. Park, J. Yoon, I. Shin, Chem. Soc. Rev. 2014, 43, 16.

[225] R. van der Meel, E. Sulheim, Y. Shi, F. Kiessling, W. J. M. Mulder, T. Lammers, Nat. Nanotechnol. 2019, 14, 1007.

[226] M. Z. El-Readi, M. A. Althubiti, J. Nanomater. 2019, 2019, DOI $10.1155 / 2019 / 4927312$.

[227] J. Yang, R. Zhang, D. C. Radford, J. Kopeček, J. Control. Release 2015, 218, 36.

[228] Y. Zhao, F. Fay, S. Hak, J. Manuel Perez-Aguilar, B. L. Sanchez-Gaytan, B. Goode, R. Duivenvoorden, C. de Lange Davies, A. Bjørkøy, H. Weinstein, et al., Nat. Commun. 2016, 7, 11221 .

[229] S. W. Morton, X. Zhao, M. A. Quadir, P. T. Hammond, Biomaterials 2014, 35, 3489.

[230] X. Hu, J. Zhang, Z. Yu, Y. Xie, J. Qi, X. Dong, Y. Lu, W. Zhao, W. Wu, NanomedNanotechnol. 2015, 11(8), 1939

[231] H. Malhaire, J. C. Gimel, E. Roger, J. P. Benoît, F. Lagarce, Adv. Drug Deliv. Rev. 2016, 106, 320.

[232] E. Roger, F. Lagarce, E. Garcion, J. P. Benoit, J. Control. Release 2009, 140, 174. 
[233] R. K. Jain, T. Stylianopoulos, Nat. Rev. Clin. Oncol. 2010, 7, 653.

[234] V. Torchilin, Adv. Drug Deliv. Rev. 2011, 63, 131.

[235] Y. Matsumura, H. Maeda, Cancer Res. 1986, 46, 6387.

[236] M. A. Radicchi, J. V. de Oliveira, A. C. P. Mendes, D. M. de Oliveira, L. A. Muehlmann, P. C. Morais, R. B. Azevedo, J. P. F. Longo, J. Mater. Chem. B 2018, 6, 7306.

[237] J. Mérian, R. Boisgard, X. Decleves, B. Thezé, I. Texier, B. Tavitian, J. Nucl. Med. 2013, 54, 1996.

[238] A. Loureiro, E. Nogueira, N. G. Azoia, M. P. Sárria, A. S. Abreu, U. Shimanovich, A. Rollett, J. Härmark, H. Hebert, G. Guebitz, et al., Colloids Surfaces B Biointerfaces 2015, 135, 90 .

[239] G. Prévot, M. Duonor-Cérutti, M. Larivière, J. Laroche-Traineau, M. J. Jacobin-Valat, P. Barthélémy, G. Clofent-Sanchez, S. Crauste-Manciet, Data Br. 2017, 15, 824.

[240] A. Karageorgis, S. Dufort, L. Sancey, M. Henry, S. Hirsjarvi, C. Passirani, J. P. Benoit, J. Gravier, I. Texier, O. Montigon, et al., Sci. Rep. 2016, 6, 1.

[241] J. F. Lovell, T. W. B. Liu, J. Chen, G. Zheng, Chem. Rev. 2010, 110, 2839.

[242] A. Ormond, H. Freeman, Materials (Basel). 2013, 6, 817.

[243] C. Park, J. Yoo, D. Lee, S. Jang, S. Kwon, H. Koo, Int. J. Mol. Sci. 2019, 20, 3958.

[244] L. A. Muehlmann, M. C. Rodrigues, J. P. F. Longo, M. P. Garcia, K. R. Py-Daniel, A. B. Veloso, P. E. N. de Souza, S. W. da Silva, R. B. Azevedo, J. Nanobiotechnology 2015, 13, 36 .

[245] R. P. A. de Matos, M. F. Calmon, C. F. Amantino, L. L. Villa, F. L. Primo, A. C. Tedesco, P. Rahal, Biomed Res. Int. 2018, 2018, 4057959.

[246] R. A. Day, D. A. Estabrook, J. K. Logan, E. M. Sletten, Chem. Commun. 2017, 53, 13043.

[247] M. Kaci, A. Belhaffef, S. Meziane, G. Dostert, P. Menu, Velot, S. Desobry, E. ArabTehrany, Colloids Surfaces B Biointerfaces 2018, 167, 165.

[248] K. Charoenkul, D. Phromyothin, in Mater. Today Proc., Elsevier Ltd, 2017, pp. 61056110.

[249] T. E. Manyarara, S. Khoza, A. Dube, C. C. Maponga, in MRS Adv., Materials Research Society, 2018, pp. 2203-2219.

[250] R. Zhang, Z. Zhang, D. J. McClements, Colloids Surfaces B Biointerfaces 2020, 194, 111202.

[251] Y. Liu, P. Le, S. J. Lim, L. Ma, S. Sarkar, Z. Han, S. J. Murphy, F. Kosari, G. Vasmatzis, J. C. Cheville, et al., Nat. Commun. 2018, 9, 4461.

[252] B. Andreiuk, A. Reisch, E. Bernhardt, A. S. Klymchenko, Chem. - An Asian J. 2019, $14,836$. 
[253] M. Jiang, X. Gu, J. W. Y. Lam, Y. Zhang, R. T. K. Kwok, K. S. Wong, B. Z. Tang, Chem. Sci. 2017, 8, 5440. 